\title{
WestVirginiaUniversity
}

THE RESEARCH REPOSITORY @ WVU

Graduate Theses, Dissertations, and Problem Reports

2017

\section{Reliability Study of Blue Phosphorescent Organic Light-Emitting Diodes}

Renyuan Yang

Follow this and additional works at: https://researchrepository.wvu.edu/etd

\section{Recommended Citation}

Yang, Renyuan, "Reliability Study of Blue Phosphorescent Organic Light-Emitting Diodes" (2017). Graduate Theses, Dissertations, and Problem Reports. 6999.

https://researchrepository.wvu.edu/etd/6999

This Thesis is protected by copyright and/or related rights. It has been brought to you by the The Research Repository @ WVU with permission from the rights-holder(s). You are free to use this Thesis in any way that is permitted by the copyright and related rights legislation that applies to your use. For other uses you must obtain permission from the rights-holder(s) directly, unless additional rights are indicated by a Creative Commons license in the record and/ or on the work itself. This Thesis has been accepted for inclusion in WVU Graduate Theses, Dissertations, and Problem Reports collection by an authorized administrator of The Research Repository @ WVU. For more information, please contact researchrepository@mail.wvu.edu. 


\title{
Reliability Study of Blue Phosphorescent Organic Light- Emitting Diodes
}

\author{
Renyuan Yang
}

Thesis submitted

to the Benjamin M. Statler College of Engineering and Mineral Resources at West Virginia University

in partial fulfillment of the requirements

for the degree of Master of Science

in

Electrical Engineering

Xian-An Cao, Ph.D., Committee Chairperson

Mark A. Jerabek, Ph.D.

Parviz Famouri, Ph.D.

Lane Department of Computer Science and Electrical Engineering

Morgantown, West Virginia 2017

Keywords: Phosphorescent, light-emitting diode, lifetime, degradation

Copyright 2017 Renyuan Yang 


\section{ABSTRACT \\ Reliability Study of Blue Phosphorescent Organic Light- Emitting Diodes}

\section{Renyuan Yang}

Organic light-emitting diodes (OLEDs) are very promising and considered as the next generation display and lighting sources due to their important merits, such as light weight, area-emission, vivid colors and flexibility. Over the last two decades, the OLED technology has drawn considerable research interest. Significant progress has been made, leading to much improved device reliability, luminance efficiency, and manufacturability. In the OLED family, phosphorescent OLEDs (PhOLEDs) are intrinsically more efficient than fluorescent OLEDs. However, compared with green and red PhOLEDs, blue PhOLEDs are considered as the 'weakest' mainly due to their low reliability, which is a great bottleneck limiting their commercial applications. Despite considerable scientific and technical efforts in recent years, the underlying mechanisms have not been fully understood and the problem remains to be solved. The goal of this work is to fabricate PhOLEDs with blue-emitting FIrpic doped in different host materials and investigate material factors contributing to fast degradation of blue PhOLEDs.

First, blue PhOLEDs based on FIrpic were fabricated by the thermal evaporation method. The performance of the PhOLEDs were optimized by tailoring key parameters including the thickness of the electron transport layer (ETL), the host material, and the doping level of the phosphorescent emitting dye within the host. In particular, through a comparative study of the PhOLEDs with four different wide bandgap host materials, we have found that the properties of the host material have a great impact on the electrical and optical characteristics of the blue PhOLEDs.

Second, stressing tests under continuous and pulsed currents were conducted to 
gain a better understanding of the factors governing the degradation process of blue PhOLEDs. Blue PhOLEDs with different hosts exhibited a lifetime varying in a wide range, suggesting a great influence of the host. By suppressing the self-heating effect through $1 \%$ pulsed current stressing, the device lifetime was improved only by 2-3.2 times. We further investigated the effects of post thermal treatment, reverse biasing, and doping concentration on the reliability of the PhOLEDs. All the findings led to the conclusion that the PhOLED degradation was mainly caused by defects generated within a narrow zone inside the emissive layer, and to achieve reliable device operation, it is vital to select an appropriate host which has good stability, enables efficient charge injection and balanced charge transport in the emissive layer. 


\section{Acknowledgements}

In the first place, I must especially thank my advisor, Prof. Xian-An Cao, for his guidance, patience and support during the past years. Owing to his support, I have opportunity to study in West Virginian University in Morgantown. He introduces me to this wonderful, promising research area of OLEDs. Moreover, he kindly provides excellent platform for me to study and do research. I cannot appreciate him too much.

I am grateful to Prof. Mark Jerabek and Parviz Famouri, who are my committee members. They give me valuable suggestion and comments on research work and help me a lot improve the thesis.

I would like to thank my lab mates, Dr. Xiaomeng Li and Mr. David A. Shelhammer, for their kindness and friendship. They offer me kind suggestion and support whenever I have problems in research or living. We discuss together, help each other. Owing to their help, I can get rich research data. I spent most wonderful days with them in Morgantown.

I would like to thank Dr. Weiqiang Ding, Kolin Brown and Marcela Redigolo of WVU Shared Research Facility (SRF) for their patient training and professional technical support.

Finally, my deepest gratitude goes to my parents and my uncle's family for their endless love and support throughout my life. I thank you all for your support and encouragement. 


\section{Publications and submitted paper}

1. X. Cao, X. Li, R. Yang, and Y. Zhou, Effects of Localized Heating at Heterointerfaces on the Reliability of Organic Light-Emitting Diodes. IEEE Electron. Dev. Lett, vol. 36 (8), 847-849 (2015).

2. X. Li, D. Shelhammer, R. Yang and X. Cao, Voltage Reduction in Organic LightEmitting Diodes by Sequential Deposition Doping. IEEE Transactions on Electron Devices. (In press)

3. R. Yang, X. Li and X. Cao, Role of wide bandgap host in the degradation of blue phosphorescent organic light-emitting diodes. Organic Electronics. (Submitted) 


\section{TABLE OF CONTENTS}

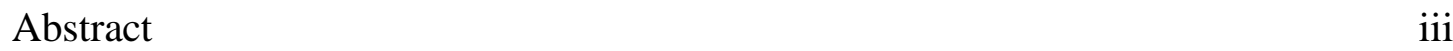

Acknowledgements $\quad$ iv

Publications and submitted paper $\quad \mathrm{v}$

Chapter 1 Introduction 1

1.1 Brief history of OLEDs 1

1.2 Working principle of PhOLEDs 2

1.2.1 Structure of OLEDs 2

1.2.2 Principle of phosphorescence in organic materials 4

1.2.3. Excitonic energy transfer and recombination in PhOLEDs 6

$\begin{array}{ll}\text { 1.3 Development and Challenges of Blue PhOLEDs } & 8\end{array}$

$\begin{array}{ll}\text { 1.3.1 Blue phosphorescent emitter } & 9\end{array}$

$\begin{array}{ll}\text { 1.3.2 Host materials for blue PhOLEDs } & 10\end{array}$

$\begin{array}{ll}\text { 1.3.3. Efficiency loss due to parasitic effects } & 11\end{array}$

$\begin{array}{ll}1.4 \text { Thesis goal and organization } & 13\end{array}$

$\begin{array}{ll}\text { References } & 13\end{array}$

Chapter 2 Fabrication of Blue PhOLEDs with Different Host Materials $\quad 17$

$\begin{array}{ll}2.1 \text { Introduction } & 17\end{array}$

$\begin{array}{ll}2.2 \text { Experimental procedure } & 18\end{array}$

$\begin{array}{ll}2.3 \text { Optimization of the baseline blue PhOLED } & 19\end{array}$

2.3.1 Organic Materials used in the baseline blue PhOLED 19

$\begin{array}{ll}\text { 2.3.2 Charge injection layers } & 20\end{array}$

2.3.3 Optimal thickness of the ETL 22

2.3.4 Optimal doping concentration of FIrpic 23

2.4 Blue PhOLEDs with different hosts 26

$\begin{array}{ll}2.5 \text { Conclusions } & 33\end{array}$

$\begin{array}{ll}\text { References } & 34\end{array}$

$\begin{array}{ll}\text { Chapter } 3 \text { Reliability Study of Blue PhOLEDs } & 36\end{array}$ 
3.1 Introduction 36

$\begin{array}{ll}3.2 \text { Experimental procedure } & 38\end{array}$

3.3 Reliability of Blue PhOLEDs with different hosts 41

$\begin{array}{ll}3.4 \text { Investigation of other degradation factors } & 47\end{array}$

$\begin{array}{ll}\text { 3.4.1 Reverse biasing } & 47\end{array}$

3.4.2 Doping concentration $\quad 48$

3.4.3 Post thermal treatment $\quad 49$

3.5 Conclusions

References $\quad 52$

Chapter 4 Conclusions and Future Work

4.1 Conclusions

4.2 Future work $\quad 56$ 


\section{Chapter 1 Introduction}

Over the last two decades, the organic light-emitting diode (OLED) technology has progressed significantly and attracted tremendous research interest. OLEDs are considered as the next generation technology for displays and solid-state lighting due to their important merits, such as light weight, area-emission, vivid colors, wide viewing angle, self-emitting, high contrast, small thickness, low operating voltage, fast switching and flexibility [1-4]. Commercial OLED products have already hit the market and are used in cell phone screens, television, computer monitors, watches and some large-area light-emitting elements [5]. However, their great potential for general lighting has not been fully fulfilled.

\subsection{Brief history of OLEDs}

OLEDs essentially rely on organic electroluminescence (EL), which is light emission from an organic material driven by the electric field. The first organic EL was reported by Bernanose in 1953 [6]. In his work, an alternating potential with a potential difference up to $2000 \mathrm{~V}$ was applied across a thin film of cellophane adsorbed with acridine derivatives. In 1982, Vincett observed organic EL from anthracene crystals with solid electrodes. EL was visible in normal room lighting with a voltage of about $30 \mathrm{~V}$ and in dark room with a voltage of only $12 \mathrm{~V}$ [7]. The breakthrough was that the driving voltage was significantly reduced less than $100 \mathrm{~V}$. However, the voltage was still too high for practical use. Organic EL did not get much scientific attention until the first OLED device was made by Tang and Van Slyke in 1987 [8]. The double-layer OLED was based on a simple p-n heterostructure prepared by thermal evaporation deposition and sandwiched between two electrodes. The device consisted of N,N'diphenyl-N,N'-bis(3-methylphenyl)1,1'-biphenyl-4,4' diamine (TPD) as the hole transport layer and tris(8-hydroxyquinoline) aluminum $\left(\mathrm{Alq}_{3}\right)$ as both the electron transport layer and the light emission layer. The driving voltage was below $10 \mathrm{~V}$, and the external quantum efficiency was $1 \%$, much higher than any before. Shortly afterwards, in 1988, the first multi-layer OLED was developed by Chihaya Adachi et 
al [9]. This achievement initiated the development of OLEDs with a high efficiency and a low driving voltage. Up to now, many OLEDs with a sophisticated, optimized structure and superior performance are based on the multi-layer prototype. In 1990, Richard Friend's group at Cambridge University prepared the first polymer OLED, which exhibited green-yellow light with efficiency about 0.05\% [10]. In 1994, the first white OLED was made by Kido et al. Three fluorescent dyes (blue, green and orange) were mixed together into a single emission layer. The white OLED was considered as an ideal candidate for a future energy-saving lighting source [11]. In 1998, Forrest and et al. discovered the triplet harvesting effect and developed the first phosphorescent OLED (PhOLED), which was based on the phosphorescent dye 2,3,7,8,12,13,17,18octaethyl-21H,23H-porphine platinum(II) (PtOEP) [12]. The PhOLED harvesting both singlet and triplet excitons showed superior efficiency over traditional fluorescent OLEDs, which harvest only singlet excitons. Since then, OLEDs have drawn growing research interests and enormous efforts have been made in this promising field.

Over the years, tremendous progress has been achieved in the OLED field, leading to greatly improved color gamut, device reliability, luminance and power efficiency [13-15]. Commercial products based on OLEDs have already been developed for flatpanel display applications. In the near future, they are expected to find widespread applications in the commercial lighting market [16].

\subsection{Working principle of PhOLEDs}

\subsubsection{Structure of OLEDs}

As a basic prototype shown in Figure 1.1(a), the first OLED utilized a bi-layer structure, consisting of hole transport layer (HTL) and electron transport layer (ETL) sandwiched between anode and cathode on a glass substrate. It is considered as the simplest and most basic architecture for the OLEDs. When an electric field is applied across the OLED, electrons are injected from the cathode into the lowest unoccupied molecular orbital (LUMO) of the ETL, and holes are injected from anode into the highest occupied molecular orbital (HOMO) of the HTL. For organic materials, it is 
widely believed that molecules with electron-donating groups usually possess the hole transport property, whereas molecules with electron-accepting moieties generally exhibit the electron transport character, and molecules with both electron-donating and accepting groups may show the bipolar carrier transport property, which is a desirable feature for the EML. Driven by electric field, the holes and electrons move through the transport layers and meet at the interface of HTL/ETL. Excitons are formed by the hole/electron recombination and then decay either radiatively to produce light or nonradiatively to generate heat.

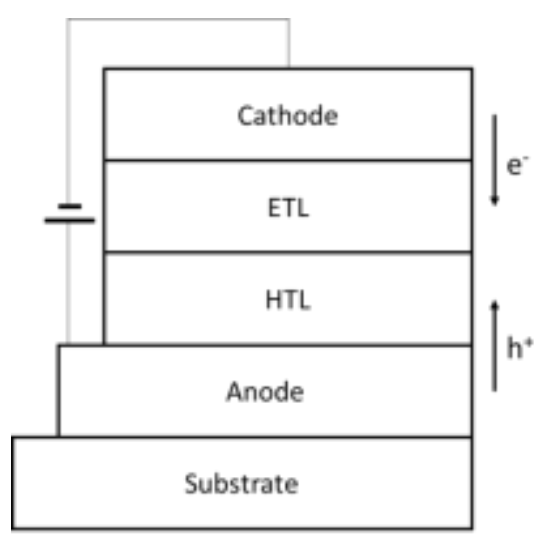

(a)

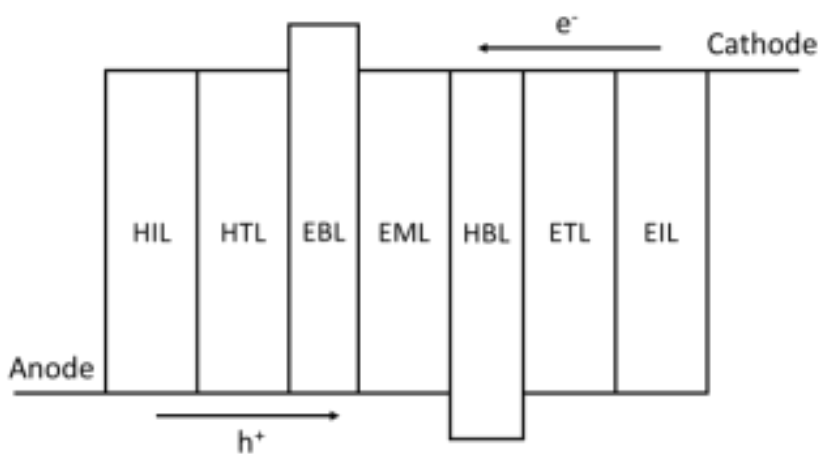

(b)

Figure 1.1 Architecture of (a) the first OLED, and (b) a state-of-the-art OLED.

To achieve high efficiency, the architecture can be more complicated. Figure 1.1(b) shows the possible functional layers that a typical OLED would have. A great advantage of the multilayer structure is that each layer can be optimized for specific purposes. The hole injection layer (HIL) and electron injection layer (EIL) help inject charge carrier from the electrodes into the transport layers, which subsequently conduct charge carriers into emission layer (EML). The transport layers are characterized by high charge carrier mobility and matched energy level to that of the EML. The EML plays the key role of an OLED. Charge recombination, exciton formation and radiation process mainly take place in the EML. Phosphorescent dye tends to show low luminance efficiency when the dye concentration is high, because of concentration quenching. Therefore, the dye needs to be doped in a host matrix, in order to avoid high concentration. As a result, in PhOLEDs, the EML is typically composed of host and 
guest material. Normally, the excitons initially form on the host molecules, followed by energy transfer from the host to the guest molecules. In order to prevent undesired carrier migration out of the EML, carrier blocking layer can be inserted between the EML and the transport layer. For instance, electron blocking layer (EBL) can restrain electron overflow from the EML, due to its higher LUMO energy level than that of the EML. The hole blocking layer plays a similar role to the EBL. Under some circumstances, an exciton blocking layer can even been utilized to confine excitons inside of the EML, because the excitons can hop from one molecule to another, only if the energy level configuration allows. Specifically for blue PhOLEDs, the triplet excitons tend to diffuse out of the EML, because the triplet energies of the host and dopant are very high $(>2.5 \mathrm{eV})$, sometimes much higher than those of the carrier transport materials. In sum, generally for a PhOLED, carrier transport layers and the EML are quite essential and indispensable, whereas the injection and blocking layers are still important but play complementary roles depending on specific requirements.

\subsubsection{Principle of phosphorescence in organic materials}

The operation principle of EL devices is based on radiative decay of excitons. Properties of the excitons primarily determine the overall performance and luminescent efficiency of the OLEDs. An exciton in organic materials may be considered as a pair of a hole and an electron. The electron is usually excited on to the LUMO energy level and a hole is created in the ground state on the HOMO level, due to the absence of the electron. The exciton formation process in OLEDs mainly takes place in the EML. The wavelength of the light emission is determined by the band gap of the emitter material, which is normally the energy difference of LUMO and HOMO. Nevertheless, the condition is a little different in the PhOLEDs. The color of phosphorescence depends on the energy of the lowest triplet excited states, because the light emission comes from radiative decay of triplet excitons. Usually, the triplet energy of the phosphor is slightly lower than the singlet. Other vibration emission peaks also accompany the triplet emission. They usually appears to have longer wavelength than the main peak. 
Normally, the vibration emission should be suppressed to maintain color purity.

Under the influence of electric field, charge carriers travel from the electrodes through aforementioned functional layers to the EML. In the EML, a pair of a hole and an electron recombines together, forming an exciton. The total spin of the two electron system may be either $S=0$ or $S=1$. The $S=0$ state is known as a singlet, and the $S=1$ is a triplet, which contains three possible states. Normally, the population ratio of singlet to triplet number is $1: 3$.

In addition, the spin of the exciton conserves during the exciton decay and therefore influences the luminance. The ground state of most molecules is a singlet state, and only singlet excited states can radiatively decay, producing light emission. Because the emission of a photon conserves spin, radiation of singlet excitons is fast and efficient. This emission is known as fluorescence. In contrast, almost all energy of the remaining triplet states is lost to non-radiative decay. The singlet states make up only a quarter of all excited states. As a consequence, the internal quantum efficiency (IQE) of fluorescent OLEDs has a maximum of 25\% [17].

However, the situation would be changed if heavy atoms are introduced in the molecules, due to the fact that heavy atoms such as platinum or iridium can enhance spin-orbit interactions within a molecule. The excited singlet and triplet states can mix together so that the triplet states possess some properties similar to the singlet. As a result, the decay of the triplet state is partially allowed. This light emission from triplet transition is known as phosphorescence. Nevertheless, the radiative decay of the triplet is still significantly slower than that of the singlet. This problem would be solved if singlet-triplet mixing in the exciton state has a faster rate in the radiative decay than in the non-radiative. For the case of the metalloorganic complexes of the $\mathrm{Pt}(\mathrm{II})$ and $\mathrm{Ir}(\mathrm{III})$, the lowest singlet excited state has rather low radiative rate for transitions to the ground state and low absorption intensity [18]. The exciton mixing can enhance intersystem crossing (ISC), leading to transition from the singlet to the triplet with high probability, because the energy of the singlet excitons is a bit higher than that of the triplet. The ISC 
process which transfers energy from the singlet to the triplet states is highly competitive with the singlet emission. Nearly all singlet excitons on the phosphor sites finally lead to triplet excitons by ISC. In this way, singlet states of emitter molecules transfer energy to the triplet, and the triplet excitons radiatively decay producing phosphorescence. Thus, phosphorescence can harvest $100 \%$ of the excitons, making a 100\% IQE [12].

A bulk layer of phosphorescent emitter exhibits a noticeable reduction in quantum efficiency. This effect is known as concentration or aggregation quenching. Improved efficiency can be achieved if the phosphorescent emitters are doped in a host material. High quantum efficiency can be obtained when the phosphorescent emitters are dispersed in the host material with doping level in the range of 1-10 wt\%.

\subsubsection{Excitonic energy transfer and recombination in PhOLEDs}

Ideally, the energy on host molecules should transfer to the phosphor, unless emission from the host is desired. In the host/guest system, the host molecule can also be called the donor, and the guest is the acceptor. There are two types of energy transfer in the EML: Förster and Dexter energy transfer. Singlet excitons on donor are mainly transferred to the accepter via Förster energy transfer mechanism, which is a type of a dipole-dipole interaction between the dipole transition moments [19-21]. The Förster transfer mechanism requires significant spectra overlap between the emission of the host material and the absorption of the dopant, as well as strong emission of the host and absorption of the dopant. This mechanism is characterized by long range: within $100 \AA$ separation between the donor and accepter. The triplet energy on the host molecule can migrate to other molecules by Dexter energy transfer. Dexter transfer strongly depends on the overlap of donor and acceptor orbitals, leading to a requirement of short-range distance $(<10 \AA)$ between the donor and accepter. The Dexter mechanism can be applied to spin-forbidden transitions, where the Förster energy transfer cannot occur. Therefore, the Dexter mechanism determines the triplet-triplet energy transfer, triplet-triplet annihilation and other spin-dependent processes. Triplet excitons migrate from site to site, ultimately locating on a phosphorescent molecule. It 
is worth noting that the Dexter transfer is responsible for the triplet-triplet annihilation, a parasitic effect of PhOLEDs.

Besides the energy transfer from the host to the guest molecules, exciton formation directly on the guest molecules is possible in the EML. In this scenario, the host material plays the role of carrier transport. For direct carrier trapping on the phosphorescent guest dopant, a significant overlap and offset of the HOMO and LUMO energies of the host and guest molecules is necessary. It is widely believed that PhOLEDs are more efficient if the excitons are directly generated on the phosphorescent emitter.

Once the triplet exciton is on the guest sites, they should be confined on the sites in order to produce light emission. These triplet excitons on the phosphor are easy to migrate to the triplet states on other molecules (including both host and guest molecules) via highly efficient Dexter type triplet-triplet energy exchange steps. The triplet excitons on the phosphor are mostly populated on the lowest triplet excited states. To prevent triplet migration out of phosphors, the lowest triplet excited states of the host molecule should desirably be higher than that of the guest. This feature in the host/guest system is important to build an efficient PhOLED.

Moreover, it should be mentioned that the location and profile of the charge recombination zone has great impact on the OLEDs performance. Exciton distribution in the EML is strongly determined by the electrical (transport) properties of the various materials used, for example, the carrier mobility, HOMO and LUMO energy levels. High luminance efficiency requires a balanced hole/electron ratio which is ideally 1:1. However, bipolar material, which is characterized by equal abilities to conduct both holes and electrons, is hard to synthesize. The hole and electrons experience different energy barriers to reach the EML. As a consequence, the excitons are usually formed in a thin slab of the EML in the proximity of an interface to an adjacent material layer. A thin recombination zone gives rise to excitons of high concentration. For PhOLEDs, high density induces concentration quenching, leading to efficiency loss. The width of the recombination zone is less than $5 \mathrm{~nm}$. Despite this fact, the EML still needs to be 
thick enough to prevent charge leakage, which may otherwise result in efficiency decrease and even undesired emission of other organic materials. A broad recombination zone is desired for high efficiency of the PhOLEDs. Up to now, it is still a challenge of achieve this goal.

The bipolar material is a key factor to solve the problem of narrow recombination zone. Although this material is not easy to synthesize, it can be obtained by mixing two host materials together: a hole-transport and an electron-transport type host material. By adjusting the ratio of these two parts or the mixing profile, a relatively balanced charge ratio can be achieved, due to balanced carrier mobility and blended energy levels configuration. Here, excitons are formed on either side of the interface between these two layers. This concept has successfully been adopted in fabrication of high efficiency white OLEDs. Ultimately, inherently bipolar materials are required and more effort is needed to develop these materials.

\subsection{Development and Challenges of Blue PhOLEDs}

The PhOLEDs are drawing much attention because they can theoretically reach 100\% IQE by harvesting both singlet and triplet excitons [12]. As an indispensable primary color of white emission, blue OLEDs have a great influence on the applications of display and lighting. The blue light can be converted to green and red colors by a color-changing medium. Compared with green and red PhOLEDs, the development blue PhOLED is less satisfactory [22-25]. Typically, the blue part is considered as 'the weakest link' in the realization of a high-efficiency white PhOLED. A lifetime of hundred thousands of hours has been achieved for green and red PhOLEDs, whereas typical blue PhOLEDs lasts only a few hours [26-28]. A great improvement in lifetime of blue PhOLEDs was recently made by Forrest et al [29]. They reported a novel blue electrophosphorescent device with a graded dopant concentration profile in a broadened emissive layer. The lifetime of the blue device was extended up to ten times. However, state-of-the-art blue PhOLEDs are still not suitable for commercial use. A significant improvement in the reliability is urgently required. The short lifetime of blue PhOLEDs 
is generally believed to be closely linked with the inherent properties of the materials in blue PhOLEDs, for instance, the wide energy bandgap and parasitic effects associated with the organic emitter and host.

\subsubsection{Blue phosphorescent emitter}

The phosphorescent emitter plays the most important role in PhOLEDs and determines the device performance, such as color, efficiency and stability. So far, efficient blue phosphors are mainly based on Iridium (Ir) complexes as they can meet several critical requirements of blue phosphorescent emitter materials: (i) a large triplet energy, (ii) a short lifetime of triplet excitons, and (iii) a high photoluminescence (PL) quantum efficiency. The triplet energy of blue phosphors should be in the range of 2.50$2.80 \mathrm{eV}$ for blue emission, corresponding to emission wavelengths in a range of 443$496 \mathrm{~nm}[30]$. Blue phosphors should have a $\mu$ s order lifetime for triplet excitons, which is required by fast radiative decay [31]. In addition, a high PL quantum efficiency is needed in order to achieve theoretical maximum internal quantum efficiency close to $100 \%[12]$.

Many excellent blue phosphorescent materials have been synthesized, for instance, iridium(III)bis(4,6-difluoro-phenylpyridinato)-3(trifluoromethyl)-5- (pyridine-2-yl)1,2,4-triazolate (FIrtaz) [32], iridium(III)bis(4,6- difluorophenylpyridinato)-(5-pyridin2-yl)-1H-terazolate (FIrN4) [32, 33], iridium(III)bis [(3,4,5-rifluorophenyl)-pyridinato$\left.\mathrm{N}, \mathrm{C}^{2}\right]$ picolinate $\quad\left(\mathrm{F}_{3}\right.$ Irpic $) \quad$ [34], Bis[2-(4,6- difluorophenyl)pyridinato- $\left.\mathrm{C}^{2}, \mathrm{~N}\right]$ (picolinato)iridium(III) (FIrpic) [35-40], iridium(III) bis(4',6'difluorophenylpyridinato)tetrakis(1-pyrazolyl)borate (FIr6) [32, 41, 42]. Among them, FIrpic is considered as the most efficient one and is also widely utilized as dopant in blue PhOLEDs. The $\mathrm{F}_{3}$ Irpic has a similar molecular structure to FIrpic. The PL quantum yield of $F_{3}$ Irpic is higher than that of FIrpic. However, emission peak of $F_{3}$ Irpic shows a red-shift of $8 \mathrm{~nm}$, compared with FIrpic. Fir6 shows improved blue color, but it has worse stability.

Despite the merits, the Ir-based blue phosphors suffer a serious weakness of 
instability and relatively low luminance efficiency, compared with green and red phosphors. The blue emitters need to have sufficiently high triplet energy to produce blue photons. Therefore, the triplet excited excitons with high energy increase the susceptibility of deterioration for the blue emitters. The low stability can be induced in another way. In order to achieve a high triplet energy, the blue phosphors usually involve strong electron-withdrawing groups, such as fluoride and cyanide. However, C-F bonds in blue phosphors are easy to break under electrical stimulation. As a result, the dopant greatly contributes to low reliability of blue PhOLEDs.

\subsubsection{Host materials for blue PhOLEDs}

For standard PhOLEDs, the phosphorescent dopant is dispersed in the host material. The host material is required to generate excitons and then efficiently transfer energy to the dopant. Moreover, it should be characterized by high stability. Endurable thermal and electrochemical stability together with stable morphology is desired to ensure a long device lifetime. Taking into account both the mechanism for exciting the triplet state of the dopant and the device structure, the requirements for making a good host material are: (i) the HOMO/LUMO energy level of the host material should envelop those of the dopant to allow direct carrier trap on the dopant species; (ii) matched HOMO/LUMO levels of the host to the adjacent carrier transport layers are needed to reduce energy barrier and thus enhance charge injection; (iii) the host material should have balanced carrier mobility to get a broad charge recombination zone; (iv) the triplet energy level of the host material should be higher than that of the guest, to ensure forward energy transfer from the host to the guest and prevent reverse transfer; (v) PL spectrum of the host should have good overlap with the absorption of the dopant, in order to facilitate Förter energy transfer; (vi) host material with good stability benefits device lifetime [20, 43].

Considering the triplet energy of blue phosphor has already been high, synthesis of host material with higher triplet energy and large band gap proves more difficult. Besides, the stability of the host material may be a problem, due to high energy excitons. 
Normally, trade-off must be made among some of the factors, because it is impossible for all the criteria to be satisfied simultaneously. For instance, usually, high triplet energy and high electron mobility cannot be achieved simultaneously in organic molecules. Because weak conjugation of molecules result in high triplet energy and low carrier mobility as well [44].

Usually, a broad charge recombination zone is difficult to achieve, due to (i) unbalanced charge ratio of the host material, and (ii) the holes and electrons experience different energy barrier to travel from the electrodes to the EML [45]. A narrow recombination zone results in high exciton density and further triplet-triplet annihilation, leading to efficiency decrease and short lifetime.

\subsubsection{Efficiency loss due to parasitic effects}

In typical PhOLEDs, the EQE decreases from its peak value with increasing current density. This efficiency drop is called the roll-off effect in PhOLEDs. This effect increases with current density and doping level. Blue fluorescent OLEDs are often used to replace blue phosphorescent OLEDs when the operating current density is high, because the former suffer less from the roll-off effect. There are mainly two major factors in PhOLEDs contributing to this effect: triplet-triplet annihilation (TTA) and triplet-polaron annihilation (TPA) [46].

The TTA is an important case of exchange energy transfer. Two triplet excited states on two molecules produce two other states. Possibly, both of the resultant states are singlet. One is on ground state, which is also a singlet state. The other one is on a higher singlet state, the energy of which is twice the triplet energy. The higher singlet state would relax to the lowest singlet state. This process can briefly be represented as: $\mathrm{T}_{1}+\mathrm{T}_{1} \rightarrow \mathrm{S}_{\mathrm{n}}+\mathrm{S}_{0} \rightarrow \mathrm{S}_{1}+\mathrm{S}_{0} . \mathrm{T}_{1}, \mathrm{~S}_{\mathrm{n}}, \mathrm{S}_{1}$ and $\mathrm{S}_{0}$ are the lowest triplet state, higher singlet state, lowest singlet and ground state, respectively. This process leads to fast decrease of triplet population and energy loss, because no light is emitted in the TTA process. Usually, the TTA is considered as the most important factor contributing to the efficiency roll-off in the PhOLEDs [47, 48]. 
TPA is another parasitic effect, leading to triplet quenching and efficiency loss. TPA is an energy transfer process that a triplet exciton transfer energy to a charged molecule (polaron), forming an excited polaron. Similar to the TTA, the TPA increases with triplet density. Under some circumstances, the TPA is proposed to be the dominant mechanism for the roll-off, instead of the TTA [49].

The parasitic effects not only play roles in efficiency roll-off, but cause degradation issues. Molecules on much higher energy levels can be generated as mediate products by both TTA and TPA processes. They are considered as 'hot molecules', which incur further reactions, fueling device degradation. For instance, direct dissociation can be induced by the highly vibrantly excited states. Intrinsic device degradation is caused by the chemical conversion of a fraction of molecules in the organic materials. Then, the reaction products can act as non-radiative recombination centers, luminescent quenchers or deep charge traps, leading to luminance loss. The defects can trap charge carriers, accelerating the TPA process. Moreover, accumulation of the charge traps in the bulk of organic layers together with defects at the layer interfaces contributes to driving voltage increase. It should be noted that a single defect site is not necessarily limited to one of the mentioned roles but might in fact act as an electron and hole-trap as well as a quenching site at the same time. The formation of these defects can be caused by different processes and proceed via a variety of possible pathways.

The parasitic effects exist in all PhOLEDs. However, the problems induced by the TTA and TPA are more serious in blue PhOLEDs [50]. High-energy intermediate states in the blue PhOLEDs often increase the accessibility of higher lying excited states leading to dissociation and bond rupture processes. Moreover, due to high triplet energies, the excess energy dissipated through TTA and TPA process in the blue PhOLEDs is significantly higher than that in green or red PhOLEDs, resulting in faster degradation and shorter device lifetime. 


\subsection{Thesis goal and organization}

As discussed above, blue PhOLEDs show considerably poorer reliability as compared with green and red ones. The goal of this thesis is to fabricate efficient blue PhOLEDs based on FIrpic and study their degradation mechanisms under high-current stressing.

Chapter 2 describes the fabrication and optimization of blue PhOLEDs with FIrpic doped in a wide bandgap host. To obtain high-efficiency blue PhOLEDs, the layer structure is optimized through comparative studies of the electrical and optical characteristics of PhOLEDs with different host materials, FIrpic doping concentrations, and electron-transport layer thicknesses.

Chapter 3 aims to investigate the device degradation mechanisms and determine the major material factors affecting the device reliability. Lifetimes of blue PhOLEDs with different hosts stressed under high currents are measured. To suppress the selfheating effect, the PhOLEDs are also stressed using 1\% pulsed currents. Furthermore, the effects of post thermal treatment, reverse biasing, and doping concentration on the blue PhOLED lifetime are studied. Based on the observations, the key host material factors which have a significant impact on the device reliability are discussed.

\section{References}

[1] S. Chen, L. Deng, J. Xie , L. Peng, L. Xie, Q. Fan, W. Huang, Adv. Mater. 2010, 22, 5227-5239.

[2] J. Park, D. Shin and S. ParkSemicond, Sci. Technol. 26 (2011) 034002.

[3] M. Katsuhara, I. Yagi, A. Yumoto, M. Noda, M. Hirai, R. Yasuda, T. Moriwaki, S. Ushikura, A. Imaoka, T. Sasaokaand K. Nomoto, Proc. of SPIE Vol. 7417 74170W1.

[4] C. D. Dimitakopoulos and P. R. L. Malenfant, Adv. Mater. 14, 99 (2002).

[5] https://www.oled-info.com/oled-tv

[6] A. Bernanose, Br. J. Appl. Phys. 6 S54 (1955). 
[7] P. S. Vincentt, W. A. Barlow, R. A. Hann, and G. G. Roberts, Thin Solid Films 94, $171(1982)$

[8] C. W. Tang and S. A. VanSlyke, Appl. Phys. Lett. 51, 913 (1987).

[9] C. Adachi, S. Tokito, T. Tsutsui and S. Saito, Jap. J. Appl. Phys. 2A, L269 (1988).

[10] J. H. Burroughes, D. D. C. Bradley, A. R. Brown, R. N. Marks, K. MacKay, R. H. Friend, P. L. Burns and A. B. Holmes, Nature 347, 539 (1990).

[11] J. Kido, K. Hongawa, K. Okuyama, K. Nagai, Appl. Phys. Lett. 64 (1994) 815817.

[12] M. A. Baldo, D. F. O'Brien, Y. You, A. Shoustikov, S. Sibley, M. E. Thompson and S. R. Forrest, Nature 395, 151 (1998).

[13] X. Yang, X. Xu and G. Zhuo, J. Mater. Chem. C, 3, 913, (2015).

[14] M. C. Gather and S. Reineke, J. Phot. Eng. 5, 057607-1, (2015).

[15] H. Jeong, H. Shin, J. Lee, B. Kim, Y. Park, K. S. Yook, B. K. An and J. Park, J. Phot. Eng. 5, 057608-1, (2015).

[16] S. Schmidbauer, A. Hohenleutner and B. Konig, Adv. Mater., 25, 2114-2129, (2013).

[17] C. Ulbricht, B. Beyer, C. Friebe, A. Winter, and U. S. Schubert, Adv. Mater., 21, 4418-4441, (2009).

[18] H. Xu, R. Chen, Q. Sun, W. Lai, Q. Su, W. Huang and X. Liu, Chem. Soc. Rev. 43, 3207-3812, (2014).

[19] S. Reineke and M. Baldo, Phys. Status Solidi A 209, No. 12, 2341-2353 (2012).

[20] B. Mi, Z. Gao, Z. Liao, W. Huang and C. H. Chen, Sci. China Chem., 53, 16791694, (2010).

[21] B. Minaev, G. Baryshnikov and H. Agren, Phys. Chem. Chem. Phys., 2014, 16, $1719-1758$.

[22] S. Xia, R. Kwong, V. Adamovich, M. Weaver and J. Brown. IEEE 07CH37867 45th Annual International Reliability Physics Symposium, Phoenix 2007, 253-257.

[23] V. Adamovich, B. D’Andrade, M. Weaver and J. Brown, Conference on Lasers and 
Electro-Optics, CLEO 2007, 1-2.

[24] V. Jarikov, J. Vargas, D. Kondakov, R. Youngh, K. Klubek, L. Liao and C. Brown Dig. Tech. Pap. - Soc. Inf. Disp. Int. Symp. 2007, 38, 1188-1192.

[25] J. Seo, S. K. Jeon, M. S. Gong, J. Y. Lee, C. H Noh and S. H. Kim, J. Mater. Chem. C, 2015, 3, 4640-4645.

[26] R. Seifert, I. R. de Moraes, S. Scholz, M. C. Gather, B. Lussem and K. Leo, Organic Electronics 14 (2013) 115-123.

[27] S. Scholz, D. Kondakov, B. Lussem and K. Leo, Chem. Rev., 115, 8449-8503, (2015).

[28] I. R. de Moraes, S. Scholz, B. Lussem and K. Leo, Organic Electronics 12 (2011) 341-347.

[29] Y. Zhang, J. Lee and S. R. Forrest, Nat. Comm. 5, 5008 (2014).

[30] K. S. Yook and J. Y. Lee, Adv. Mater. 24,3169-3190, (2012).

[31] C. Ulbricht, B. Beyer, C. Friebe, A. Winter, and U. S. Schubert, Adv. Mater., 21, 4418-4441, (2009).

[32] S. Yeh, C. Chen, Y. Song and M. Ho, Journal of the Society for Information Display 13(10):857-862.

[33] T. Tsuboi, H. Murayama, S. Yeh, M. Wu and C. Chen, Optical Materials 31 (2008) 366-371.

[34] S. Lai, S. Tao, M. Chan, M. Lo, T. Ng, S. Lee, W. Zhao and C. Lee, J. Mater. Chem., 2011, 21, 4983.

[35] S.-J. Su, E. Gonmori, H. Sasabe, J. Kido, Adv. Mater. 20 (2008) 4189-4194.

[36] H. Sasabe, E. Gonmori, T. Chiba, Y.-J. Li, D. Tanaka, S.-J. Su, T. Takeda, Y.-J. Pu,K.-i. Nakayama, J. Kido, Chem. Mater. 20 (2008) 5951-5953.

[37] S.H. Ye, Y.Q. Liu, J.M. Chen, K. Lu, W.P. Wu, C.Y. Du, Y. Liu, T. Wu, Z.G. Shuai, G.Yu, Adv. Mater. 22 (2010) 4167-4171.

[38] R. Holmes, S. Forrest, Y. Tung, R. Kwong, J. Brown, S. Garon and M. Thompson, Appl. Phys. Lett., 82, 2003,2422. 
[39] S. Yoo, J. Yoon, N. Kim, J. Kim, J. Kang, C. Moon and W. Kim, Journal of Luminescence, 160, (2015) 346-350.

[40] J. Wang, H. Zhang, W. Ji, H. Zhang and F. Zhu, Jpn. J. Appl. Phys. 54, 101601 (2015).

[41] C. Adachi, R. C. Kwong, P. Djurovich, V. Adamovich, M. A. Baldo, M. E. Thompson, S. R. Forrest, Appl. Phys. Lett. 2001, 79, 2082-2084.

[42] R. J. Holmes, S. R. Forrest, Y. J. Tung, R. C. Kwong, J. J. Brown, S. Garon, M. E. Thompson, Appl. Phys. Lett. 2003, 82, 2422-2424. [43] F. Li, H. Tang, J. Anderegg, J. Shinar, Appl. Phys. Lett. 1997,70,1233.

[44] H. Tang, F. Li, J. Shinar, Appl. Phys. Lett. 1997, 71, 2560.

[45] H. W. Choi, S. Y. Kim, W.-K. Kim, J.-L. Lee, Appl. Phys. Lett. 2005, 87,082102.

[46] H. W. Choi, S. Y. Kim,W.-K. Kim, K. Hong, J.-L. Lee, Appl. Phys. Lett. 2006, 100, 064106.

[47] A. Quan, F. Teng, Z. Xu, D. Wang, S. Yang, Y. Hou, Y. Wang, Phys. Lett. 352- 434 (2006).

[48] Michele Sessolo and Henk J. Bolink, Adv. Mater. 23, 1829-1845 (2011).

[49] H. J. Bolink, H. Brine, E. Coronadoa and M. Sessolo, J. Mater. Chem., 20, 40474049 (2010).

[50] E. J. W. List, C. H. Kim, J. Shinar, A. Pogantsch, G. Leising and W. Graupner, Appl. Phys. Lett. 76, 2083 (2000). 


\section{Chapter 2 Fabrication of Blue PhOLEDs with Different Host Materials}

\subsection{Introduction}

For phosphorescent organic light-emitting diodes (PhOLEDs), the emission layer (EML) is the most vital part. The phosphorescent dye is characterized by concentration quenching effect. Usually, the dye is dispersed in a host material with low doping level, to avoid quenching [1-5]. As a result, the EML of PhOLEDs is composed of host and guest material.

Much work done was focused on the development of blue phosphorescent emitters with improved efficiency and color purity. The development of high energy or deep blue phosphorescent materials has been one of the major challenges in this area. The design and preparation of blue phosphorescent complexes with high triplet energy level, high emission quantum yield and good stability are far more difficult to be realized than those of red and green phosphorescent complexes. Among the blue phosphorescent dyes, Bis[2-(4,6-difluorophenyl)pyridinato- $\left.\mathrm{C}^{2}, \mathrm{~N}\right]$ (picolinato)iridium(III) (FIrpic) and iridium(III)bis(4',6'-difluorophenylpyridinato)tetrakis(1-pyrazolyl)borate (FIr6) are considered as excellent dopants for blue PhOLEDs [6-8]. Although Fir6 shows bluer color, it suffers worse lifetime. In comparison, FIrpic is much favorable.

The selection of host materials is of great importance for the preparation of efficient PhOLEDs. Many key properties must be considered. For example, the singlet and triplet levels of the host should be higher than those of phosphorescent dopants to enable efficient energy transfer. High thermal stability is needed to improve the operation stability of devices. Blue PhOLEDs with various host materials have been demonstrated. For instance, Lee and et al. doped FIrpic in 1,3bis(triphenylsilyl)benzene (UGH3) host [9]. They also used 1,3-bis(Ncarbazolyl)benzene (mCP) as the host material [10]. The blue PhOLEDs with mCP showed external quantum efficiency of $\sim 9 \%$. Chopra and et al. reported high efficiency 
and low roll-off for blue PhOLEDs based on FIrpic doped in a mixed host, which consisted of di-[4-(N,N-ditolyl-amino)-phenyl]cyclohexane (TAPC) and 2,8bis(diphenylphosphoryl)dibenzothiophene (PO15) [11].

In this chapter, blue PhOLEDs with an EML comprising FIrpic doped in various host materials are fabricated by vapor deposition. The device performance is optimized by tailoring of the ETL thickness and FIrpic doping concentration. The electrical and optical characteristics of PhOLEDs with different hosts are measured and compared.

\subsection{Experimental procedure}

The PhOLEDs were fabricated on glass substrates with pre-patterned indium tin oxide (ITO), of which the sheet resistance was $\sim 15 \Omega / \square$. The substrates were cleaned with acetone, methanol and deionized water, and dried in air before they were treated with $\mathrm{O}_{2}$ plasma for $5 \mathrm{~min}$. They were then transferred to a thermal evaporation chamber, which was maintained in high-vacuum condition $\left(<1 \times 10^{-6}\right.$ torr $)$ by a cryopump. The organic layers were deposited at a pre-calibrated rate $\sim 0.1 \mathrm{~nm} / \mathrm{s}$. An aluminum cathode was deposited through a shadow mask. The thickness and deposition rate of each layer were measured by quartz crystal monitors. All the layers were deposited without breaking vacuum. The active device area was $0.1 \mathrm{~cm}^{2}$. Four identical devices were fabricated on each glass substrate. After deposition of all layers was completed, the devices were transferred in $\mathrm{N}_{2}$-filled glovebox, where the ambient was free from $\mathrm{O}_{2}$ and water moisture. They were encapsulated with epoxy and a glass lid. All the devices were characterized in room temperature.

The electroluminescence spectra were measured with Ocean Optics fiber-optic spectrometer. The current-voltage and luminance-current data were measured with an Agilent 4156C semiconductor parameter analyzer and a computer controlled Keithley 238 high current source measure unit, respectively. To calculate the external quantum efficiency (EQE), the OLEDs were placed directly onto the surface of a calibrated silicon photodetector ( $1 \mathrm{~cm}$ in diameter). Photoluminescence spectra were measured by 
Hitachi F-7000 spectrophotometer. Absorption and transmission data were collected by Hitachi U-3900H spectrophotometer.

\subsection{Optimization of the baseline blue PhOLED}

\subsubsection{Organic Materials used in the baseline blue PhOLED}

FIrpic is used as emitter for blue PhOLEDs, due to its high efficiency. In 2005, Kawamura et al. showed that photoluminescence quantum efficiency of FIrpic could reach nearly $100 \%$ when doped in host material with high triplet energy [12]. FIrpic is characterized by high triplet energy band gap $(2.62 \mathrm{eV})$, which responds to desirable blue emission.

Figure 2.1 shows the chemical structures of organic materials used in the baseline blue PhOLED. N,N'-Di(1-naphthyl)-N,N'-diphenyl-(1,1'-biphenyl)-4,4'-diamine (NPB) and bathophenanthroline (BPhen) are used as hole transport layer (HTL) and electron transport layer (ETL), respectively, due to their high carrier mobility [13, 14]. To ensure sufficient energy transfer, $\mathrm{mCP}$ is selected as the host material for FIrpic, because of its high triplet energy $(2.9 \mathrm{eV})$. Due to its higher hole mobility than electron mobility, mCP is considered as hole transport host material [15].

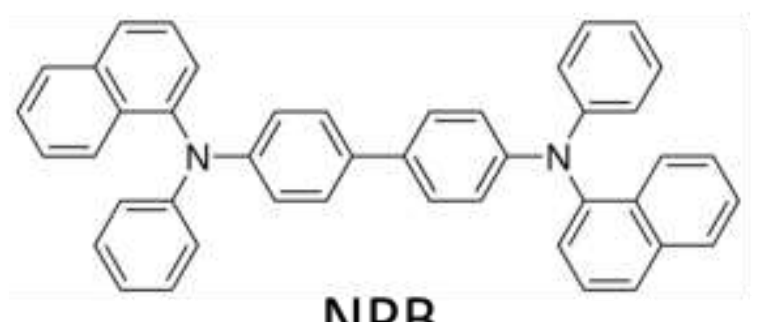

NPB<smiles></smiles>

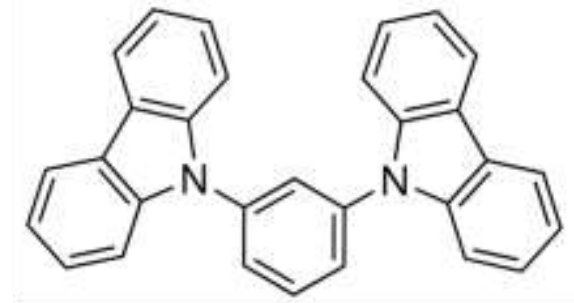

$\mathrm{mCP}$

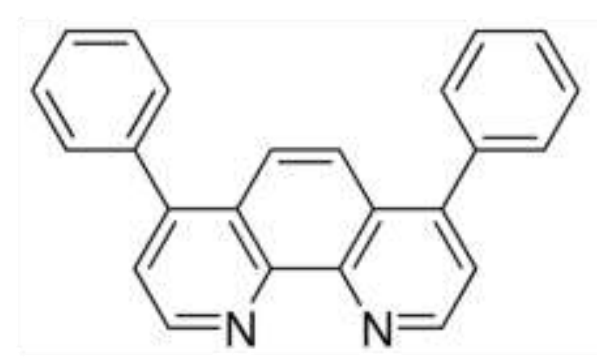

BPhen

Figure 2.1 Chemical structures of NPB, mCP, FIrpic and BPhen. 


\subsubsection{Charge injection layers}

A thin layer of molybdenum trioxide $\left(\mathrm{MoO}_{3}\right)$ is added between ITO and NPB, to serve as hole injection layer (HIL), as shown in Figure 2.2. Many reports showed that $\mathrm{MoO}_{3}$ interlayer could enhance hole injection and hence increase current density by reducing the energy barrier [16-18].

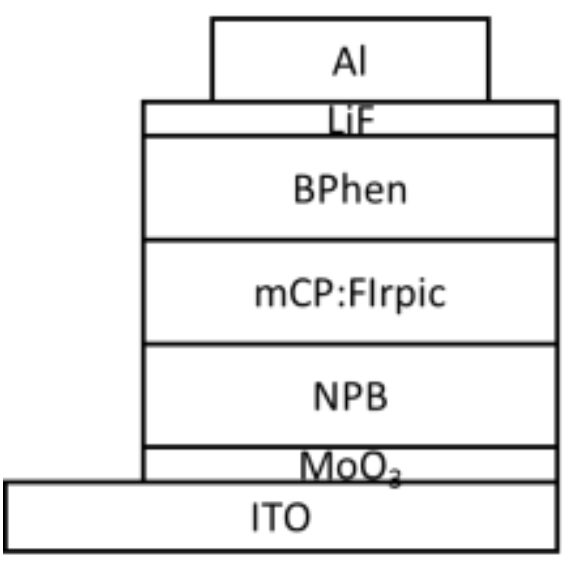

(a)

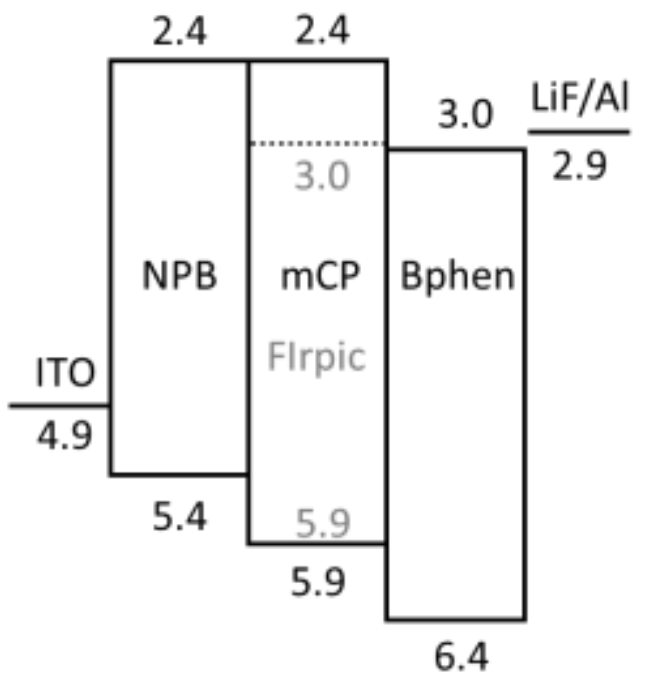

(b)

Figure 2.2 (a) Layer structure of blue PhOLED. (b) Schematic energy level diagram of blue PhOLED.

Reliability comparison between devices with and without $\mathrm{MoO}_{3}$ layer is shown in Figure 2.3. Device with $\mathrm{MoO}_{3}$ interlayer has better durability than that that without $\mathrm{MoO}_{3}$, due to three functions of the $\mathrm{MoO}_{3}$ interlayer: (i) reduce energy barrier, (ii) isolate the ITO from the NPB and (iii) reduce ITO roughness. Large amount of Joule heating can be generated at the interface with a high energy barrier and consequently cause problems to the local organic molecules, such as decomposition and aggregation. A thin layer of $\mathrm{MoO}_{3}$ attenuates such an energy barrier. The $\mathrm{MoO}_{3}$ interlayer isolates the ITO from the HTL, preventing possible atom migration. For example, indium atoms driven by electrical field can migrate into the organic layers, which provide quenching sites in the EML. In our experiment, the ITO is treated with oxygen plasma prior to deposition of each layer. After the treatment, oxygen atoms are forced on the ITO surface and thus unstable. During operation, the ITO loses the oxygen, which may react 
with radical NPB molecules. Moreover, losing oxygen atoms inevitably cause the work function of the ITO to decrease. The oxygen diffusion can be alleviated by introducing a $\mathrm{MoO}_{3}$ interlayer. Dark spots partially relate to the ITO roughness. Because, during operation, ITO spikes give rise to higher local voltage than the surrounding area, leading to more joule heating around the spikes. Liu demonstrated that $\mathrm{MoO}_{3}$ deposition could improve the ITO roughness. The thickness of the $\mathrm{MoO}_{3}$ layer in our samples is only $0.5 \mathrm{~nm}$, which is much less than that in the report [19]. Therefore, the roughness improvement is not as pronounced as what was in Liu's report. Roughness improvement may play only a minor role in this scenario.

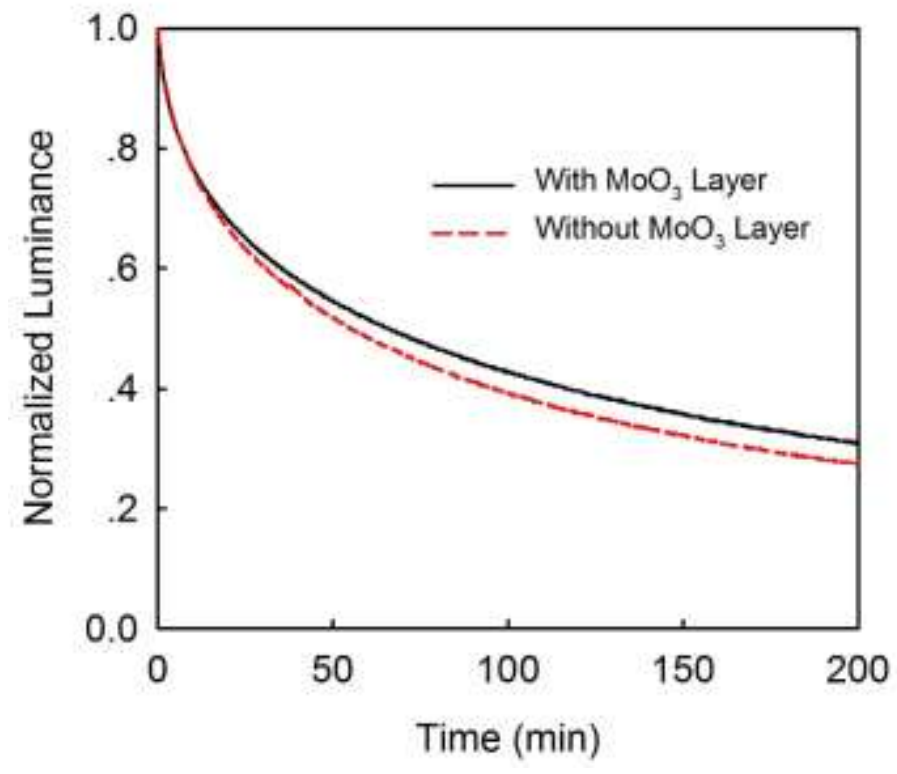

Figure 2.3 Normalized luminance decay curves of devices with and without $\mathrm{MoO}_{3}$ interlayer.

On the cathode side, efficient electron injection requires cathode with a low barrier, matching the LUMO level of the organic material, which is usually $2-3 \mathrm{eV}$. Some metal with a low work function such as $\mathrm{Ca}$ and $\mathrm{Mg}$ are used as the cathode [20, 21]. Nevertheless, they are unstable, because they are very sensitive to moisture and oxygen. $\mathrm{Al}$ is a popular cathode material, due to its stability and low cost. A thin layer $(<1 \mathrm{~nm})$ of lithium fluoride $(\mathrm{LiF})$ capped with $\mathrm{Al}$ layer is widely utilized as the cathode to enhance electron injection. For the LiF/Al cathode, many mechanisms are proposed to explain the electron injection enhancement, such as tunneling effect, band bending at 
the metal/organic interface, the formation of interfacial dipoles or chemical dissociation of the LiF layer during Al evaporation [22].

For the reasons above, in our growth, $\mathrm{LiF}$ is inserted between $\mathrm{BPhen}$ and $\mathrm{Al}$ to function as electron injection layer (EIL). The LiF/Al cathode has matched work function $(\sim 2.9 \mathrm{eV})$ to lowest unoccupied molecular orbitals (LUMO) energy level of the ETL more than the pure Al cathode $(\sim 4.2 \mathrm{eV})$, resulting in lower ETL/cathode energy barrier, as indicated in Figure 2.2(b).

\subsubsection{Optimal thickness of the ETL}

The ETL has great impact on OLED performance. Large ETL thickness results in series resistance increase. For the point view of energy consumption and power efficiency, thinner ETL is favorable. However, more factors should be taken into account before the optimal thickness is determined. For instance, the ETL thickness also affects the escaping light from the anode side. All the layers are sandwiched between anode and cathode. All the visible light escapes from the transparent ITO anode. A part of the light rays hit the opaque but reflective Al layer and bounce back. The direct and reflected rays interfere with each other [23]. The distance between the recombination zone and the cathode determines whether the interference is constructive or destructively by changing the optical path difference between the direct and the reflected waves. The ETL has even more complicated impact on the OLEDs. For example, change in the ETL ingredient or thickness leads to the change of electric field distribution throughout the device structure. Since the carrier mobility has strong relation to the electric field, the carrier mobility changes in response to varying ETL thickness and hence affects the electron/hole ratio in the EML, which result in a shift of charge recombination zone and ultimately influence the performance of the device.

To investigate the influence of the ETL on the blue PhOLED performance, devices with different ETL thickness are fabricated with the structure of ITO $(100 \mathrm{~nm}) / \mathrm{MoO}_{3}$ (0.5 nm) / NPB (40 nm) / mCP:FIrpic (10 wt\%) (30 nm) / BPhen (x nm) / LiF (0.5 nm) / $\mathrm{Al}(100 \mathrm{~nm})$, as shown in Figure 2.2(a). In this case, BPhen is used as the ETL, of 
which the thickness varies ( $x=25 \mathrm{~nm}, 35 \mathrm{~nm}, 45 \mathrm{~nm}, 55 \mathrm{~nm}$, respectively). Figure 2.4(a) illustrates current density-voltage (J-V) characteristics of the PhOLEDs with different BPhen thickness. At $100 \mathrm{~mA} / \mathrm{cm}^{2}$, the voltages of the devices with BPhen thickness of 25, 35, 45 and $55 \mathrm{~nm}$ are 12.2, 13.1, 13.4 and $13.7 \mathrm{~V}$, respectively. The voltage increases with increasing BPhen thickness, simply because of the increase of series resistance. Inevitably, the device with $25 \mathrm{~nm}$ has the lowest operating voltage. Figure 2.4(b) shows luminance-current density (L-J) characteristics of devices with different BPhen thickness. The device with $45 \mathrm{~nm}$ has the best brightness, which is attributed to the best balanced electron/hole ratio as well as constructive wave interference.

From the perspective of lowering applied voltage, the optimal thickness is $25 \mathrm{~nm}$. But the $25 \mathrm{~nm}$ device suffers the lowest brightness, according the L-J characteristics. In contrast, the device with $45 \mathrm{~nm}$ has moderate $\mathrm{J}-\mathrm{V}$ curve and the best L-J characteristics. Overall, the device with BPhen of $45 \mathrm{~nm}$ thickness has the best performance.

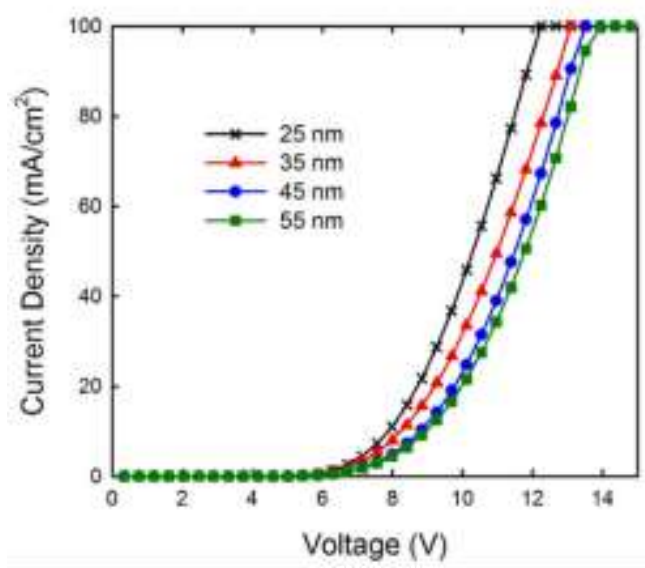

(a)

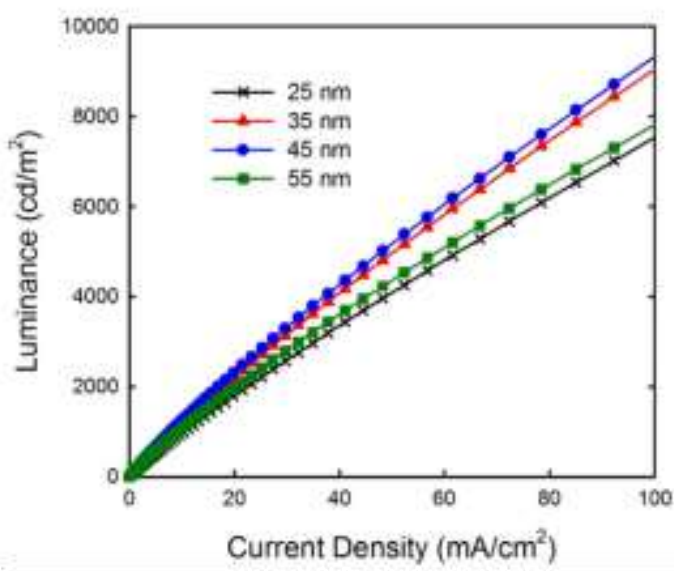

(b)

Figure 2.4 (a) Current density-voltage and (b) luminance-current density characteristics of blue PhOLEDs with different BPhen thickness.

\subsubsection{Optimal doping concentration of FIrpic}

Blue PhOLEDs with different host/guest weight ratios have been prepared with the structure of ITO $(100 \mathrm{~nm}) / \mathrm{MoO}_{3}(0.5 \mathrm{~nm}) / \mathrm{NPB}(40 \mathrm{~nm}) / \mathrm{mCP}:$ FIrpic $(\mathrm{x} \mathrm{wt} \%)$ $(30 \mathrm{~nm}) / \mathrm{BPhen}(45 \mathrm{~nm}) / \mathrm{LiF}(0.5 \mathrm{~nm}) / \mathrm{Al}(100 \mathrm{~nm})$, as shown in Figure 2.2(a). The 
phosphorescent material, FIrpic, is evenly doped in the host material, mCP. The weight ratio of the dopant and host material varies from 4 to $20 \mathrm{wt} \%$.

Figure 2.5(a) shows the J-V curve of PhOLEDs with different weight ratios. The driving voltage monotonically decreased with increasing weight ratio. Because FIrpic molecules facilitates the electron injection. The energy level diagram of the PhOLED is shown in Figure 2.2(b). LUMO energies of mCP, FIrpic, BPhen are $2.4 \mathrm{eV}, 3.0 \mathrm{eV}$ and $3.0 \mathrm{eV}$, respectively. Electrons need to overcome an energy barrier of $0.6 \mathrm{eV}$ to travel from BPhen to $\mathrm{mCP}$ molecules. In contrast, there is matched LUMO levels between FIpric and BPhen. As the FIrpic concentration increases, the electron injection from the ETL to the EML becomes more efficient. Since mCP is hole transport host material, FIrpic molecules improve charge ratio by facilitating electron injection [24].

Figure 2.5(b) shows the driving voltages required to maintain a current density of $100 \mathrm{~mA} / \mathrm{cm}^{2}$. The operating voltage shows initial fast drop followed by slow decline, implying saturated improvement on electron injection.

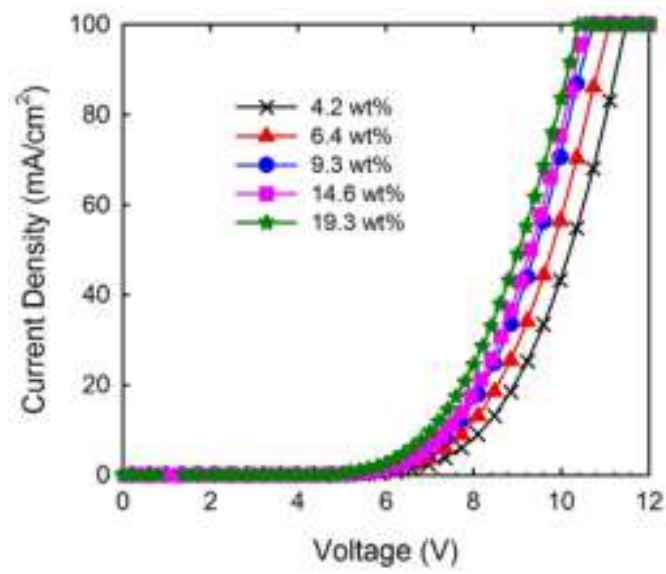

(a)

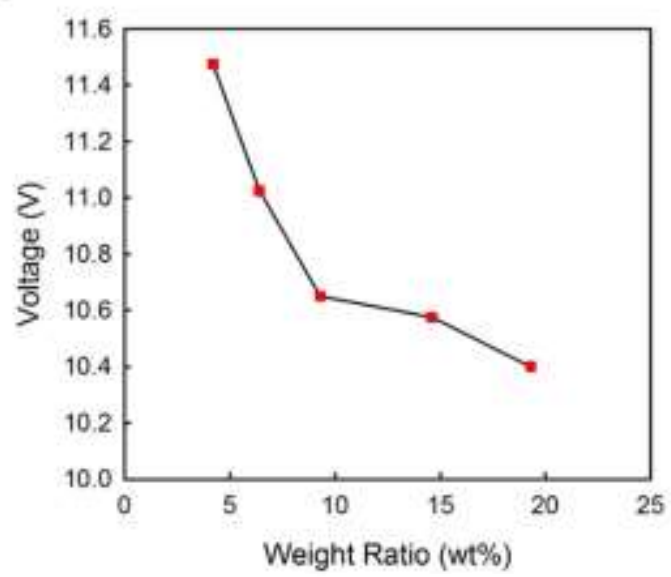

(b)

Figure 2.5 (a) Current density-voltage characteristics of blue PhOLEDs with different weight ratios. (b) Operating voltage of devices with different weight ratios when the current density was $100 \mathrm{~mA} / \mathrm{cm}^{2}$.

Figure 2.6(a) illustrates the comparison of L-J characteristics of PhOLEDs with different weight ratios. Figure 2.6(b) shows the brightness as a function of dopant concentration with current density of $20 \mathrm{~mA} / \mathrm{cm}^{2}$. The brightness of the PhOLEDs 
increases with weight ratio of FIrpic to $\mathrm{mCP}$ when it is lower than $10 \mathrm{wt} \%$. However, similar L-J curves indicate saturated brightness when the weight ratio is above $10 \mathrm{wt} \%$. The PhOLED is based on guest/host system. A small part of excitons are formed directly on FIrpic molecules, whereas most of excitons are firstly on the host species, followed by energy transfer from host to guest molecules. In the low doping level region $(<10 \mathrm{wt} \%)$, the brightness has strong relation to FIrpic concentration, mainly due to (i) more energy acceptors and emitters, and (ii) improved charge ratio. In the high doping level region, the PhOLEDs suffer two pronounced parasitic effects: triplet-triplet annihilation (TTA) and triplet-polaron annihilation (TPA). The higher concentration of the exciton on the phosphorescent molecules, the severer are the TTA and TPA effects $[24,25]$. Therefore, the PhOLEDs with high doping level suffer low efficiency. The parasitic effects may be compensated by improved charge ratio, resulting in saturated luminance.

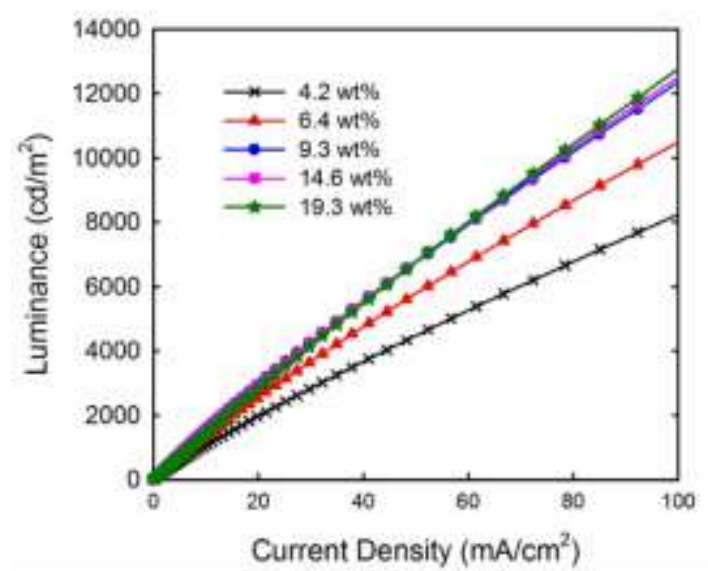

(a)

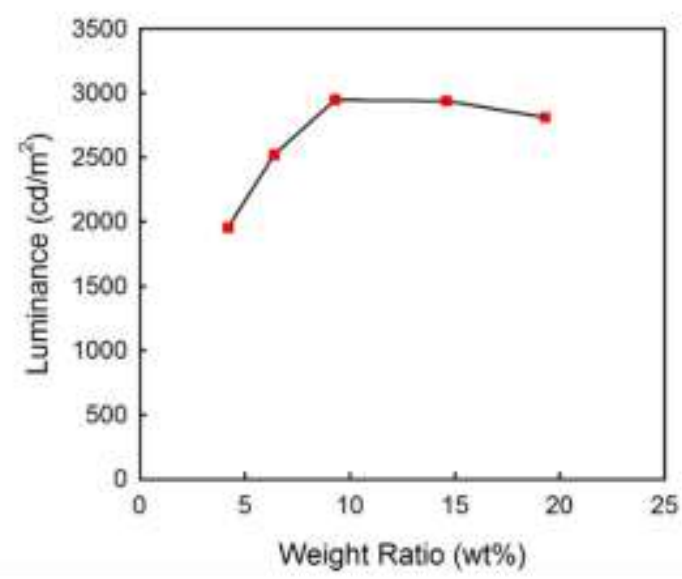

(b)

Figure 2.6 (a) Luminance-current density characteristics and (b) luminance at $20 \mathrm{~mA} / \mathrm{cm}^{2}$ of blue

\section{PhOLEDs with different weight ratios.}

Figure 2.7 shows the EQE characteristics of blue PhOLEDs with different weight ratios. All the devices exhibit decreasing EQE (the efficiency roll-off) when the current density is high. This phenomenon is explained by the TTA and TPA process, because the exciton population on FIrpic increases with current density. As Figure 2.7 indicates, the device with concentration of $9.3 \mathrm{wt} \%$ has the highest EQE. Doping concentration 
higher than $10 \mathrm{wt} \%$ improves $\mathrm{J}-\mathrm{V}$ curves and shows saturated luminance. However, high doping concentration would increase the cost. Because FIrpic is rather expensive, due to extreme rarity of Ir element. As a result, the optimal weight ratio of FIrpic to $\mathrm{mCP}$ is about $10 \mathrm{wt} \%$.

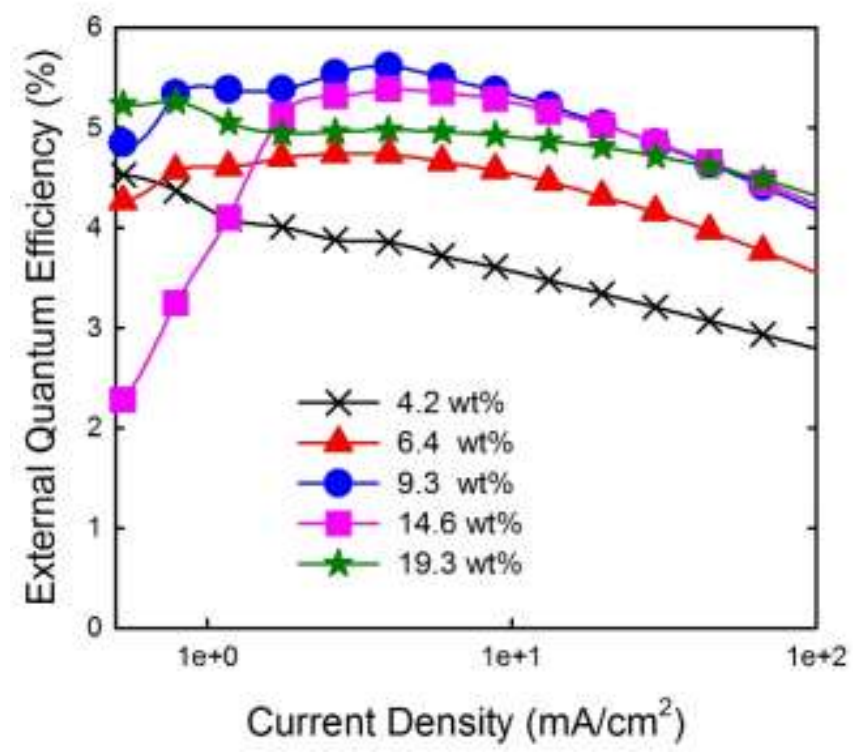

Figure 2.7 External quantum efficiency of blue PhOLEDs with different weight ratios.

\subsection{Blue PhOLEDs with different hosts}

Besides $\mathrm{mCP}$, there are a few other organic materials with large band gap that can be used as host for FIrpic. 4,4'-bis(N-carbazolyl)-1,1'-biphenyl (CBP) is popular host material for red and green PhOLEDs, due to satisfying triplet energy confinement and high carrier mobility. But it seems not suitable for blue phosphors. As shown in Table 2.1, the triple energy of $\mathrm{CBP}$ is $2.56 \mathrm{eV}$, which is lower than the triplet energy of FIrpic $(2.62 \mathrm{eV})$. If CBP is used as the host for FIrpic, the energy on FIrpic molecules can transfer back to CBP molecules. Tris(4-carbazoyl-9-ylphenyl)amine (TCTA) is hole transport host material, having large triplet energy $(2.85 \mathrm{eV})$ and high glass transition temperature $\left(\mathrm{T}_{\mathrm{g}}=151^{\circ} \mathrm{C}\right)$ [26]. TCTA has a high hole mobility, whereas its electron mobility is rather low. UGH3 is considered as slightly electron transport host material, though there is lack of its mobility data. It has very large triplet energy $(3.5 \mathrm{eV})$ but 
relatively low $\mathrm{T}_{\mathrm{g}}\left(46^{\circ} \mathrm{C}\right)$ [27]. In addition to host material, UGH3 can also be utilized as hole blocking material, because of its deep highest occupied molecular orbitals (HOMO) energy level $(7.2 \mathrm{eV})$ and very low hole mobility. The chemical structures of the host materials are shown in Figure 2.8.

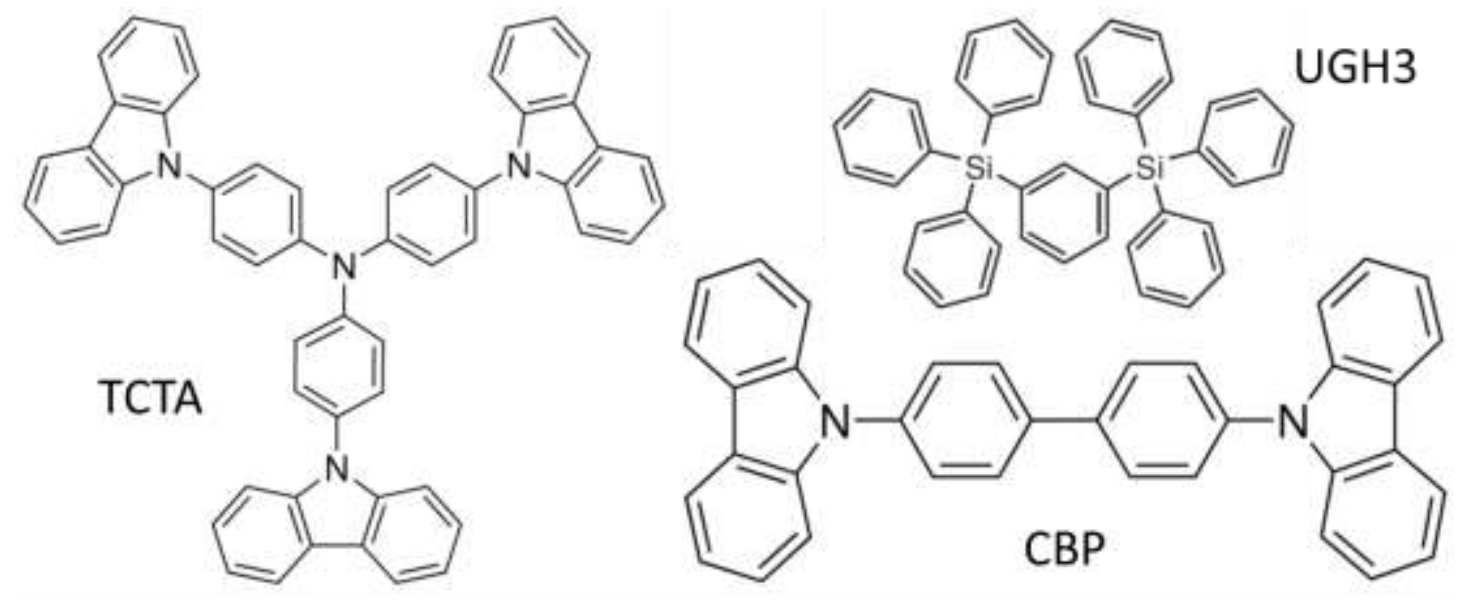

Figure 2.8 Chemical structures of TCTA, CBP, UGH3.

Blue PhOLEDs with different hosts have been fabricated, for which the structure is ITO $(100 \mathrm{~nm}) / \mathrm{MoO}_{3}(0.5 \mathrm{~nm}) / \mathrm{NPB}(40 \mathrm{~nm}) /$ Host:FIrpic $(10 \mathrm{wt} \%)(30 \mathrm{~nm}) /$ BPhen $(45 \mathrm{~nm}) / \mathrm{LiF}(0.5 \mathrm{~nm}) / \mathrm{Al}(100 \mathrm{~nm})$, as shown in Figure 2.9(a). ITO, $\mathrm{MoO}_{3}$, NPB, FIrpic, BPhen, LiF, Al is utilized as the anode, HIL, HTL, dopant, ETL, EIL, cathode, respectively. The host material is selected from one of the candidates: TCTA, CBP, mCP and UGH3. The dopant is FIrpic with a fixed weight ratio of $10 \mathrm{wt} \%$. The energy level diagram is illustrated in Figure 2.9(b).

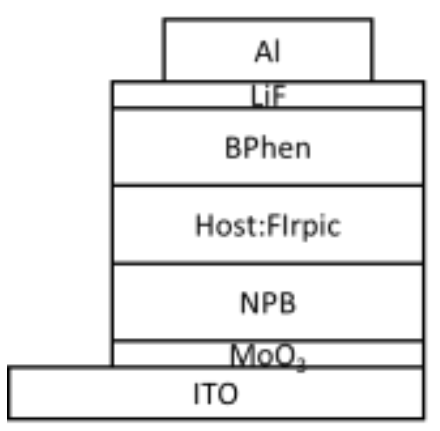

(a)

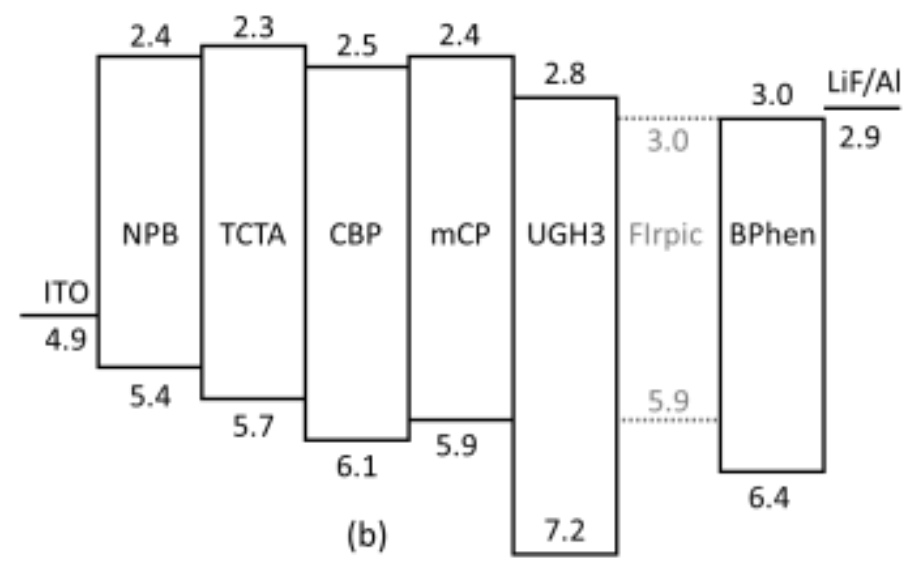

Figure 2.9 (a) Layer structure of blue PhOLEDs with different host materials (TCTA, CBP, mCP, UGH3). (b) Schematic energy level diagram of blue PhOLEDs. 
Table 2.1. Energy levels, glass transition temperature and carrier mobility of transport materials, host materials and the dopant.

\begin{tabular}{|c|c|c|c|c|c|c|}
\hline $\begin{array}{l}\text { Organic } \\
\text { Material }\end{array}$ & $\begin{array}{c}\text { HOMO } \\
(\mathrm{eV})\end{array}$ & $\begin{array}{c}\text { LUMO } \\
(\mathrm{eV})\end{array}$ & $\begin{array}{c}\text { Triplet } \\
\text { Energy } \\
(\mathrm{eV})\end{array}$ & $\begin{array}{c}\text { Glass } \\
\text { transition } \\
\text { temperature } \\
\left({ }^{\circ} \mathrm{C}\right)\end{array}$ & $\begin{array}{c}\text { Hole } \\
\text { Mobility } \\
\left(\mathrm{cm}^{2} \mathrm{~V}^{-1} \mathrm{~s}^{-1}\right)\end{array}$ & $\begin{array}{l}\text { Electron } \\
\text { Mobility } \\
\left(\mathrm{cm}^{2} \mathrm{~V}^{-1} \mathrm{~s}^{-1}\right)\end{array}$ \\
\hline TCTA & 5.7 & 2.3 & 2.85 & 151 & $3.0 \times 10^{-4}$ & $<10^{-8}$ \\
\hline $\mathrm{CBP}$ & 6.1 & 2.5 & 2.56 & 62 & $2.0 \times 10^{-3}$ & $3.0 \times 10^{-4}$ \\
\hline $\mathrm{mCP}$ & 5.9 & 2.4 & 2.9 & 55 & $3.2 \times 10^{-4}$ & $2.0 \times 10^{-4}$ \\
\hline UGH3 & 7.2 & 2.8 & 3.5 & 46 & - & - \\
\hline FIrpic & 5.9 & 3.0 & 2.62 & - & - & - \\
\hline NPB & 5.4 & 2.4 & 2.3 & 95 & $8.8 \times 10^{-4}$ & - \\
\hline BPhen & 6.4 & 3.0 & 2.5 & - & - & $5.2 \times 10^{-4}$ \\
\hline
\end{tabular}

Figure 2.10 shows the J-V characteristics of blue PhOLEDs with different hosts. At a current density of $100 \mathrm{~mA} / \mathrm{cm}^{2}$, the PhOLEDs with host of TCTA, CBP, mCP and UGH3 have voltages of 7.0, 11.8, 10.3, and 16.7 V, respectively. From Figure 2.9(b) and Table 2.1, it can be calculated that the energy barrier between HOMO levels of NPB and the hosts (TCTA, CBP, mCP and UGH3) is $0.3,0.7,0.5$ and $1.8 \mathrm{eV}$, respectively. On the other hand, the energy barrier between LUMO levels of BPhen and the host materials (TCTA, CBP, $\mathrm{mCP}$ and $\mathrm{UGH} 3$ ) is $0.7,0.5,0.6$ and $0.2 \mathrm{eV}$, respectively. The device with UGH3 has the largest operating voltage, compared with devices of other hosts, mainly because the energy barrier (HOMO difference between NPB and UGH3) is considerably high $(1.8 \mathrm{eV})$, whereas the NPB/TCTA energy barrier is only $0.3 \mathrm{eV}$. Moreover, TCTA enjoys high hole mobility. Therefore, the device with TCTA has the lowest operating voltage. 


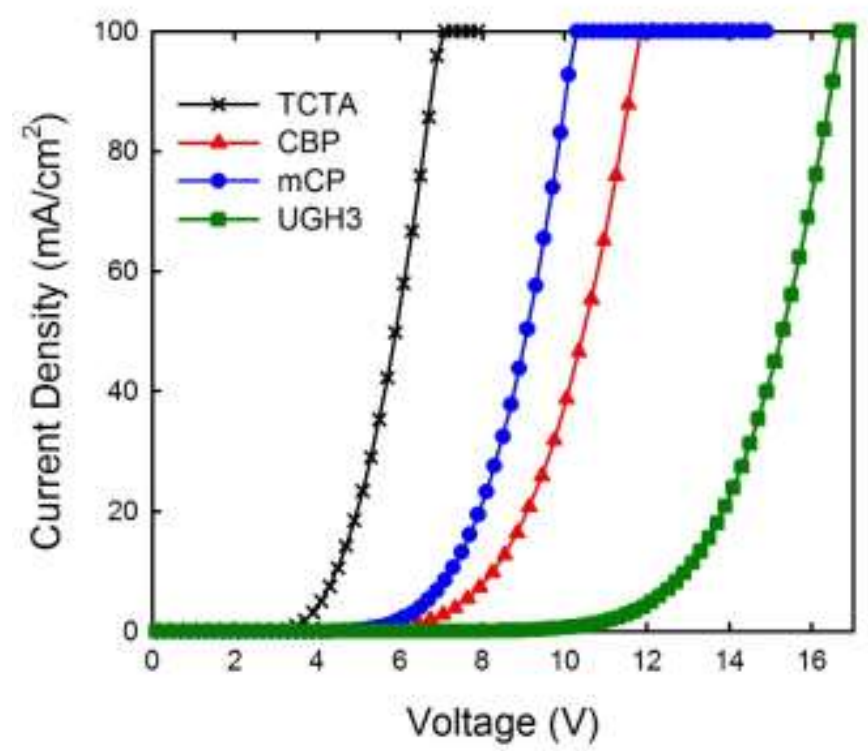

Figure 2.10 Current density vs. voltage characteristic curves of blue PhOLEDs with different hosts.

Figure 2.11 illustrates the absorption spectrum of FIrpic and normalized photoluminescence (PL) spectra of different host materials. All the spectra were recorded with the target material dissolved in chloroform. As seen, the PL emission bands of all the host materials overlap well with the absorption band of FIrpic, indicating possible efficient energy transfer in the host-guest system [28].

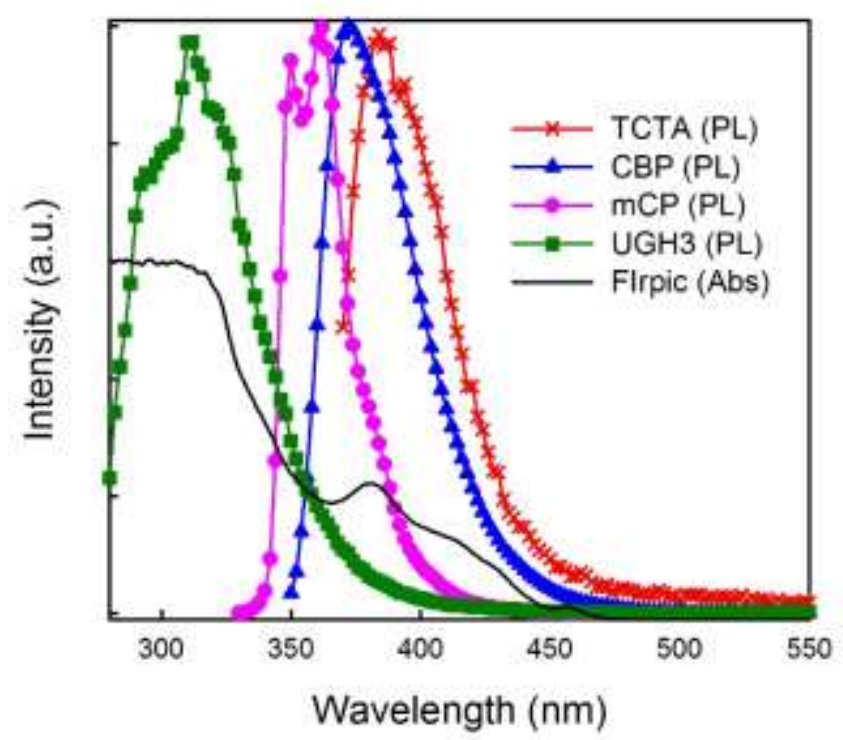

Figure 2.11 Photoluminescence spectra of the host materials (TCTA, CBP, mCP and UGH3) and the absorption spectrum of FIrpic. 
Comparison of luminance efficiency is shown in Figure 2.12. At $100 \mathrm{~mA} / \mathrm{cm}^{2}$, the luminance values of the PhOLEDs with TCTA, CBP, mCP and UGH3 are 4105, 7372, 13839 and $2145 \mathrm{~cd} / \mathrm{m}^{2}$, respectively. Among them, the PhOLED with mCP appears to be the brightest and have the highest EQE, because of its good triplet exciton confinement and balanced hole/electron ratio. CBP has smaller triplet energy $(2.56 \mathrm{eV})$ than FIrpic (2.62 eV), as shown in Table 2.1. As a consequence, triplet confinement of CBP to FIrpic would be insufficient, because energy on FIrpic molecules would transfer back to CBP, resulting in energy and efficiency loss. Despite the fact that TCTA has higher triplet energy $(2.85 \mathrm{eV})$, the TCTA device suffers low EQE. The hole mobility of TCTA $\left(\sim 3.0 \times 10^{-4} \mathrm{~cm}^{2} \mathrm{~V}^{-1} \mathrm{~s}^{-1}\right)$ is orders of magnitude larger than its electron mobility $\left(<10^{-8} \mathrm{~cm}^{2} \mathrm{~V}^{-1} \mathrm{~s}^{-1}\right)$ [29]. The ability of TCTA to conduct electrons is extremely weak, compared with that to conduct holes, leading to unbalanced electron/hole ratio in the EML. In the PhOLEDs with host of UGH3, the charge ratio is rather unbalanced, because of two reasons: (i) the UGH3 is a type of electron transport host material, and (ii) the holes need to overcome a considerable energy barrier to travel from NPB to UGH3 $(1.8 \mathrm{eV})$. Therefore, devices with hosts of TCTA and UGH3 show less efficiency, compared with CBP and mCP. It is worth noting that blocking layer was not introduced in any of the PhOLEDs, which would otherwise effectively confine host triplet excitons and charge carriers within the EML and lead to higher radiative efficiencies.

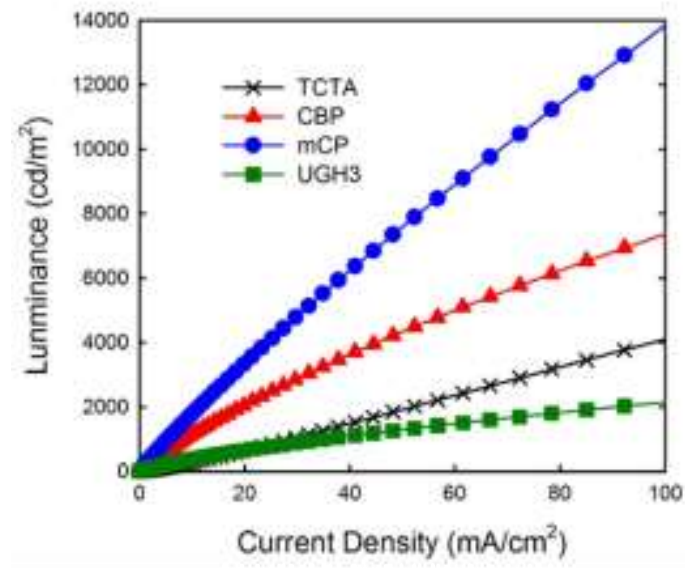

(a)

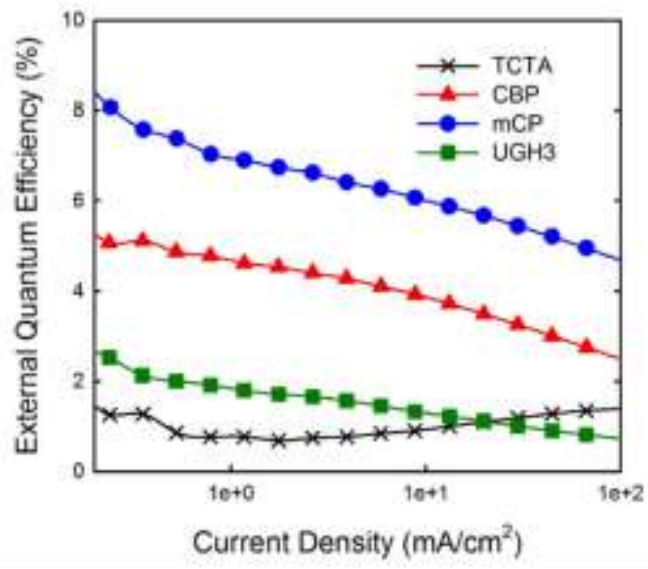

(b)

Figure 2.12 (a) Luminance-current density characteristics and (b) external quantum efficiency 
vs. current density curves of blue PhOLEDs with FIrpic doped in one of the host materials:

\section{TCTA, CBP, mCP and UGH3.}

Figure 2.13 shows normalized electroluminescence (EL) spectra of blue PhOLEDs with different hosts. The spectra were measured under room temperature when the device was operating with a current density of $100 \mathrm{~mA} / \mathrm{cm}^{2}$. All the PhOLEDs exhibit typical blue-green light with two main peaks at $\sim 474 \mathrm{~nm}$ and $\sim 502 \mathrm{~nm}$, respectively, which are the characteristic emission peaks of FIrpic. However, the PhOLEDs with different host materials vary in green/blue peak ratio. The peak ratios of the PhOLEDs with TCTA, CBP, mCP and UGH3 are 1.04, 1.14, 1.14 and 1.21, respectively. It has been found that at the $474 \mathrm{~nm}$ blue peak decreases when the recombination zone shifts towards the anode side due to of the interference effect [24]. Therefore, the difference in the peak ratio would be explained by different locations of the recombination zone in these devices.

The UGH3 device has the lowest peak at $474 \mathrm{~nm}$, indicating that the charge recombination in UGH3 device takes place near the anode most, consistent with its characteristic of an electron transport host material. As discussed above, the electron mobility of UGH3 is larger than its hole mobility. The holes accumulate at the NPB/EML interface, due to large energy barrier $(1.8 \mathrm{eV})$. The electrons need to travel through the UGH3 layer to recombine with the holes on the other side. As a result, the recombination zone would be narrow in width in the EML close the NPB/EML interface. Wang described the effect of the HTL on the efficiency of the PhOLEDs. It was reported that the HTL with low triplet energy can quench the PL of the phosphor emitter, due to energy transfer from the emitter to the triplet state of the HTL [30]. As shown in Table 2.1, the triplet energy of NPB $(2.3 \mathrm{eV})$ is much lower than that of UGH3 $(3.5 \mathrm{eV})$. The triplet excitons populated on UGH3 species may transfer energy to the triplet states of NPB molecules, leading to efficiency loss. This energy transfer to NPB may be worsened by the fact that the main recombination zone is near NPB/EML interface. Thus, the triplet exciton leakage and unbalanced charge ratio jointly contribute to low 
efficiency of the UGH3 device.

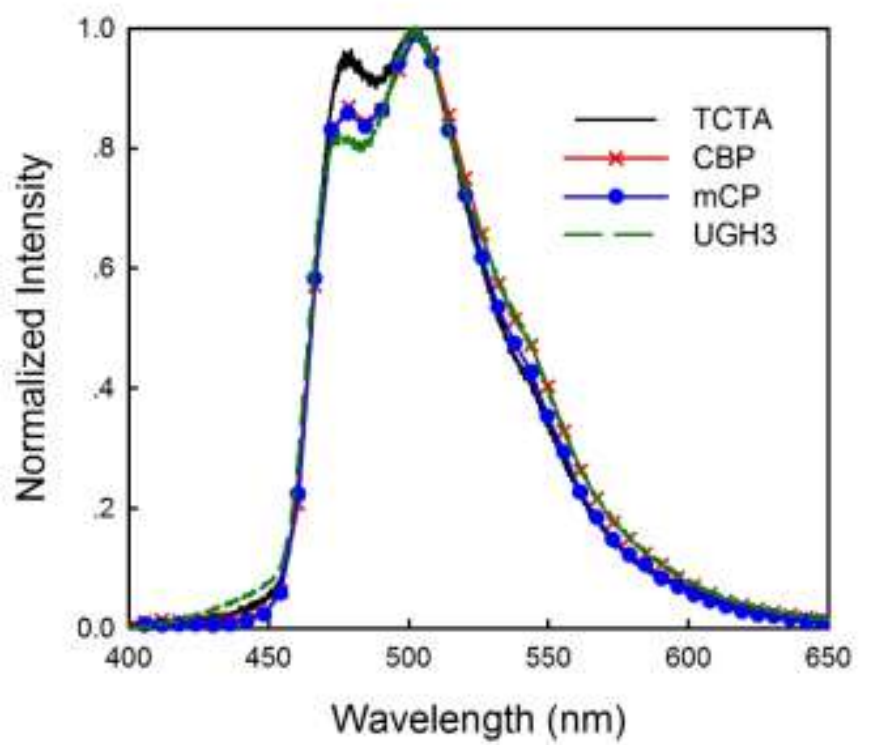

Figure 2.13 Electroluminescence spectra of blue PhOLEDs with different hosts.

TCTA has hole mobility of $3.0 \times 10^{-4} \mathrm{~cm}^{2} \mathrm{~V}^{-1} \mathrm{~s}^{-1}$ and electron mobility of less than $1 \times 10^{-8} \mathrm{~cm}^{2} \mathrm{~V}^{-1} \mathrm{~s}^{-1}$. In the TCTA device, electrons stack near EML/BPhen interface, not only because of low electron mobility, but because of large energy barrier (LUMO difference between TCTA and BPhen) $(0.7 \mathrm{eV})$. For the similar reason to the case of UGH3, recombination zone in the TCTA device is near the EML/ETLinterface, leading to low green/blue peak ratio. Also, the TCTA device suffers triplet exciton leakage from the EML to the ETL. Because the triple energy of BPhen is $2.5 \mathrm{eV}$, which is lower than that of TCTA $(2.85 \mathrm{eV})$.

In contrast to TCTA and $\mathrm{UGH} 3$, , both $\mathrm{CBP}$ and $\mathrm{mCP}$ have more comparable hole and electron mobilities as well as comparable energy barriers for electron and hole injection. Therefore, the PhOLEDs with a $\mathrm{CBP}$ or $\mathrm{mCP}$ host have a broader recombination zone, which is also located close to the ETL as they both favor hole transport.

It should be noted that the blue PhOLEDs with different hosts show difference in EL spectra in a wavelength range from $420 \mathrm{~nm}$ to $460 \mathrm{~nm}$. The EL spectrum of UGH3 device obviously exhibits a broad shoulder emission before $450 \mathrm{~nm}$, attributing to NPB 
emission [31]. This emission implies electron leakage, due to two factors: (i) UGH3 is an electron transport host material, and (ii) the energy barrier of LUMO levels of NPB and UGH3 is small $(0.4 \mathrm{eV})$, insufficient to block excess electrons. In contrast, the NPB emission cannot be detected in the device with CBP or mCP host, indicating good electron blocking property. Nevertheless, there is a weak emission between 420 and $460 \mathrm{~nm}$ in TCTA device, which should not be attributed to NPB emission. Since the electron mobility of TCTA is extremely low, very few electrons can penetrate the EML consisting of TCTA. Considering the fact that the recombination zone is near the EML/ETL interface, the small emission shoulder may originate from the broad emission band of TCTA/BPhen exciplex, of which the peak is $\sim 460 \mathrm{~nm}$.

\subsection{Conclusions}

The baseline blue PhOLED with FIrpic doped in an $\mathrm{mCP}$ host was fabricated. Nanometer-thick $\mathrm{MoO}_{3}$ and $\mathrm{LiF}$ layers were inserted between the electrodes and organic structure to enhance hole and electron injection, respectively. The thickness of the ETL was tailored in order to obtain the highest light output and the optimal thickness was found to be $\sim 45 \mathrm{~nm}$.

Blue PhOLEDs with FIrpic doped in four different host materials were fabricated and compared. The PL spectra of all the hosts overlapped the absorption spectrum of FIrpic, indicating good energy transfer from the host to FIrpic. PhOLEDs with mCP had the highest luminance and the highest external quantum efficiency due to the combined effect of matched energy level alignment, good triplet energy confinement and balanced electron/hole transport. At $20 \mathrm{~mA} / \mathrm{cm}^{2}$, the voltage was $7.9 \mathrm{~V}$, and a luminance of $3320 \mathrm{~cd} / \mathrm{m}^{2}$ was obtained. In contrast, the PhOLEDs with a TCTA or UGH3 host exhibited low luminance and efficiency mainly due to unbalanced charge injection and transport within the EML. 


\section{References}

[1] D. S. Ginley and C. Bright, MRS Bull. 25 (2000) 15.

[2] J. H. Yu and G. M. Choi, Sensors and Actuators B: Chem. 72 (2001) 141.

[3] K. L. Chopra, P. D. Paulson, and V. Dutta, Prog. Photovolt. Res. Appl. 12 (2004) 69.

[4] P. Görrn, M. Sander, J. Meyer, M. Kröger, E. Becker, H. H. Johannes, W. Kowalsky, and T. Riedl, Adv. Mater. 18 (2006). 738.

[5] V. Wood, M. J. Panzer, J. E. Halpert, J. M. Caruge, M. G. Bawendi, and V. Bulovic, ACS Nano 3 (2009) 3581.

[6] H. Guo, H. B. Andagana, and X. A. Cao, J. Electron. Mater. 39 (2010) 494.

[7] X. A. Cao, Z. Y. Jiang, and Y. Q, Zhang, Org. Electron. 12 (2011) 306.

[8] T. Minami, H. Sonohara, S. Takata, and H. Sato, Jap. J. Appl. Phys. 33 (1994) L1693.

[9] J. Lee, J. Lee, K. Song, S. J. Lee and H. Y. Chu, Appl. Phys. Lett. 92, 203305, (2008).

[10] J. Lee, J. Lee, K. Song, S. J. Lee and H. Y. Chu, Appl. Phys. Lett. 92, 133304, (2008).

[11] N. Chopra, J. S. Swensen, E. Polikarpov, L. Cosimbescu, F. So and A. B. Padmaperuma, App. Phys. Lett., 97, 033304, (2010).

[12] Y. Kawamura, K. Goushi, J. Brooks, J. J. Brown, H. Sasabe and C. Adachi, Appl. Phys. Lett. 86, 071104, (2005).

[13] E. Çetinörgü, S. Goldsmith, and R. L. Boxman, Thin Solid Films 515 (2006) 880.

[14] V. K. Jain, P. Kumar, M. Kumar, P. Jain, D. Bhandarid, and Y. K. Vijay, J. Alloys Compounds 509 (2011) 3541.

[15] D. W. Lane, J. A. Coath, K. D. Rogers, B. J. Hunnikin, H. S. Beldon, Thin Solid Films 221 (1992) 262.

[16] M. Asghar, H. Noor, M. Awan, S. Naseem, M. Hasan, Mater. Sci. in Semicond. Proc. 11 (2008) 30.

[17] M. Imada, A. Fujimori, Y. Tokura, Rev. Mod. Phys. 70 (1998) 1039.

[18] M.B. Salamon, Rev. Mod. Phys. 73 (2001) 583.

[19] S.W. Liu, Y. Divayana, X.W. Sun, Y. Wang, K.S. Leck, and H.V. Demir, Optics 
Express, 5, 4513, (2011).

[20] T.Y. Chu, J.-F. Chen, S.-Y. Chen, C.-J. Chen, C.H. Chen, Appl. Phys. Lett. 89 (2006) 053503.

[21] X.Y. Jiang, Z.L. Zhang, J. Cao, M.A. Khan, K.-U. Haq, W.-Q. Zhu, J. Phys. D Appl.Phys. 40 (2007) 5553.

[22] C.C. Chang, J.F. Chen, S.W. Hwang, C.H. Chen, Appl. Phys. Lett. 87 (2005) 253501.

[23] G. Liu, Y. Liu, B. Li and X. Zhou, J. Appl. Phys. 117, 214505, (2015).

[24] T. L. Chiu and P. Y. Lee, Int. J. Mol. Sci., 13, 7575-7585, (2012).

[25] J. Meyer, T. Winkler, S. Hamwi, S. Schmale, H.-H. Johannes, T. Weimann, P. Hinze, W. Kowalsky, T. Riedl, Adv. Mater. 20 (2008) 3839.

[26] J. Meyer, S. Hamwi, T. Bülow, H.-H. Johannes, T. Riedl, W. Kowalsky, Appl. Phys.Lett. 91 (2007) 113506.

[27] S. Tokito, K. Noda, Y. Taga, J. Phys. D Appl. Phys. 29 (1996) 2750.

[28] F. Guo, D. Ma, Appl. Phys. Lett. 87 (2005) 173510.

[29] C.W. Chen, Y.-J. Lu, C.-C. Wu, E.H.-E. Wu, Y. Yang, Appl. Phys. Lett. 87 (2005) 241121.

[30] Y. Wang, Appl. Phys. Lett. 85, 4848-2850, (2004).

[31] J. Lee, J. Lee, K. Song, S. J. Lee and H. Y. Chu, Appl. Phys. Lett. 92, 203305, (2008). 


\section{Chapter 3 Reliability Study of Blue PhOLEDs}

\subsection{Introduction}

Organic light-emitting diodes (OLEDs) are promising for flat-panel displays and solid-state lighting [1-5]. They have drawn much attention in the scientific world as well as in the industry. However, poor reliability, especially a relatively short lifetime, of OLEDs is one of the critical factors limiting commercial applications of the OLED products [6]. The lifetime of OLEDs is defined as the time lapsed for the OLED brightness to decrease to half of its initial value at a constant current or voltage [7]. In the past decade, the OLED lifetime has been improved greatly, leading to emerging commercial OLED displays used in televisions and smartphones.

Despite the success in the display field, there are more stringent requirements for the application of OLEDs in lighting, which usually needs device to operate with much higher brightness. Normally, the brighter is the OLED, the faster the OLED degrades, and the shorter is its lifetime [7]. In spite of long-term endeavor, up to now, the stateof-the-art OLEDs cannot satisfy the requirements of lighting applications [8]. Therefore, more research work needs to done to understand the degradation mechanisms and improve the lifetime of OLEDs.

For blue phosphorescent OLEDs (PhOLEDs), the issue of lifetime becomes more serious [9-12]. Compared with red and green PhOLEDs, the blue PhOLEDs have a much shorter lifetime no more than a few hours [7]. This is in part due to large energies of excitons and charge carriers in blue PhOLEDs, which can induce electrochemical reactions and molecule dissociation. The short lifetime prevents the blue PhOLEDs from applications in display and lighting. Recently, Forrest et al. provided a way to extend the lifetime of blue PhOLEDs by tenfold, making blue PhOLEDs more close to commercial use [8]. However, the improved lifetime is still far from what is needed for practical use. 
The degradation mechanism has been studied intensively and many methods have been proposed to circumvent the problems. There are various factors causing the OLEDs degrading, depending on the materials used and device architecture. Therefore, the degradation mechanisms are complex and different from one case to another. However, they can generally be categorized as extrinsic and intrinsic factors.

The extrinsic factors are caused by the external environment, for instance, preexisting particles on the substrate and presence of external species (oxygen and water moisture) from the ambient condition [13-15]. Nowadays, ultrasonic washing of substrates and encapsulation under nitrogen ambience have become a common practice and these extrinsic factors have been suppressed to a large extent [7].

Besides the extrinsic factors, the OLEDs suffer intrinsic ones, which are more challenging to investigate and control. Many causes of OLED degradation have been proposed, for example, thermal stability, interfacial degradation, traps and quenching sites. In addition, joule heating, sometimes considered as self-heating, is also believed to play an important role in device degradation $[7,16]$. The heat is generated in the bulk of organic layers as well as at the interfaces.

In blue PhOLEDs, the degradation mechanisms may be more complex, partially because organometallic molecules are used as phosphorescent emitters and thus the chemistry in PhOLEDs is a little different from conventional fluorescent OLEDs [17]. Moreover, blue PhOLEDs require a host material with a wide bandgap, resulting in a high operating voltage. So, it is possible that self-heating may be a serious issue. As discussed in Chapter 1, stronger parasitic effects can be expected in blue PhOLEDs, which can generate high energy states on organic molecules and greatly increases vulnerability of the organic materials [18]. Therefore, a lot of effort has been devoted to synthesis of blue phosphorescent emitters with high efficiency and good stability. Besides FIrpic, blue emitters including iridium(III)bis(4',6'-difluorophenylpyridinato) tetrakis(1-pyrazolyl)borate (FIr6) and iridium (III) tris[3-(2,6-dimethylphenyl)-7methylimidazo[1,2-f]phenanthridine] $\left(\operatorname{Ir}(\mathrm{dmp})_{3}\right)$ have also been used to make blue 
PhOLEDs $[8,19]$. Most previous reliability studies have focused on the degradation of these emitters [20,21], but the role of the host material in device degradation has not been fully studied yet.

In this chapter, the blue PhOLEDs based on FIrpic fabricated in Chap. 2 are subjected to constant continuous-wave and pulsed current stressing, and the evolution of their voltage and luminance is examined. The role of the host material in device degradation is investigated through a comparative study of the devices only differing in the host material. The effects of reverse biasing, doping concentration and post thermal treatment on the blue PhOLED reliability are also investigated.

\subsection{Experimental procedure}

Blue PhOLEDs have structure of ITO / $\mathrm{MoO}_{3} / \mathrm{N}, \mathrm{N}$ '-Di(1-naphthyl)-N,N'diphenyl-(1,1'-biphenyl)-4,4'-diamine (NPB) / Host : bis[2-(4,6-difluorophenyl) pyridinato- $\left.\mathrm{C}^{2}, \mathrm{~N}\right]$ (picolinato) iridium(III) (FIrpic) / bathophenanthroline (BPhen) / LiF / Al. The host material is selected from one of the four organic materials: (i) tris(4carbazoyl-9-ylphenyl)amine (TCTA); (ii) 4,4'-bis(N-carbazolyl)-1,1'-biphenyl (CBP); (iii) 1,3-bis(N-carbazolyl)benzene (mCP); (iv) 1,3-bis(triphenylsilyl)benzene (UGH3). All the host materials are characterized by large band gap [22-25]. The emission layer is $30 \mathrm{~nm}$ in thickness and the weight ratio of the dopant to the host is fixed at $10 \%$. The PhOLEDs were fabricated on glass substrates with pre-patterned ITO. The substrates were ultrasonically cleaned in individual solvents (acetone, methanol and deionized water), and dried in air. The substrates were treated with $\mathrm{O}_{2}$ plasma for 5 min before being loaded into a thermal evaporation chamber, in which each layer was deposited. During the thermal deposition process, the chamber pressure was maintained lower than $1 \times 10^{-6}$ torr by a cryopump. All the layers were deposited without breaking vacuum. The thickness and deposition rate of each layer were monitored by quartz crystal sensors. The deposition rate was kept around $0.1 \mathrm{~nm} / \mathrm{s}$. A shadow mask was used to define the shape of the cathode. The active area of an OLED was $0.1 \mathrm{~cm}^{2}$. Four identical 
OLEDs were fabricated on each substrate. After deposition of all layers was completed, the devices were transferred to a $\mathrm{N}_{2}$-filled glovebox, in which the devices were annealed and encapsulated with epoxy and a glass lid. All the devices were characterized at room temperature.

The electroluminescence (EL) spectra were measured with an Ocean Optics fiberoptic spectrometer. The current-voltage characteristics of blue PhOLEDs were recorded by an Agilent 4156C semiconductor parameter analyzer. To measure the device lifetime, an as-fabricated OLED was stressed at a constant current density and luminance data were collected periodically by a calibrated silicon photodetector. The circuit shown in Fig. 3.1 was used to measure device lifetime under pulsed stimulation. An Agilent 8114A pulse generator was used as the pulsed voltage source. The voltage on the circuit was measured by an Agilent DSO6014A oscilloscope. A variable resistor with large value is vital to the circuit and has three functions. First, the effective resistance of the OLED would be very small, only if the OLED is burnt out or experiences catastrophic failure. Therefore, a large resistor can protect the voltage generator from high current, in case of device failure. Second, the resistor combined with an oscilloscope provides a window to monitor the current level in the circuit. The third function, which is the most important, is to maintain a relatively constant current. Under electrical stressing, the effective series resistance of the OLED increases over time. Hence, given a constant pulsed voltage source, the current in the circuit would decrease over time during the operation. A large resistor is connected with the OLED in series. Therefore, the resistance increment on the OLED is negligible, compared with the overall resistance in the circuit. In this way, the current would be maintained at relatively constant level. 


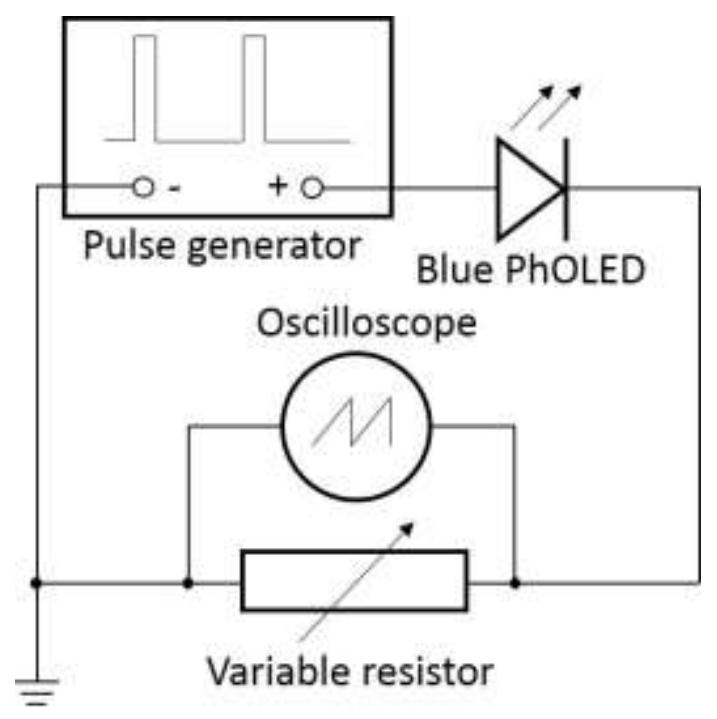

Figure 3.1. Schematic diagram of electric circuit.

When the blue PhOLEDs were stressed under pulsed voltage, the luminance was measured by an Ocean Optics fiber-optic spectrometer. The measurement was realized by calculating the intensity integration over a time period. The device was on during the pulse active time and was turned off during the rest of the period. At a fixed duty cycle of $1 \%$, the dependence of the EL intensity on pulse frequency and current density is shown in Figure 3.2. The host material of PhOLEDs is mCP. The EL intensity maintains at a constant value in low-frequency domain and drops gradually to zero at high frequency. At $5 \mathrm{~mA} / \mathrm{cm}^{2}$, the EL intensity begins dropping at $5 \mathrm{~Hz}$. The $3 \mathrm{~dB}$ bandwidth is about $125 \mathrm{~Hz}$. The dropping frequency and bandwidth increases with operating current level. At $100 \mathrm{~mA} / \mathrm{cm}^{2}$, the dropping frequency and the $3 \mathrm{~dB}$ bandwidth are 60 and $1888 \mathrm{~Hz}$, respectively. This behavior can be explained by characteristic transient electrophosphorescence, which is characterized by delay time, rise and decay time $[26,27]$. In general, the background carrier concentrations are low in organic materials. Charge carriers take time to travel from the electrodes to the EML to participate recombination, resulting in delay. The delay time depends on carrier mobility. Since the carrier mobility in organic material has an exponential dependence on the square root of the electric field, high voltage results in high carrier mobility and thus a short delay time. The rise time increases with the capacitance of the device, which is inversely proportional to the applied voltage. 


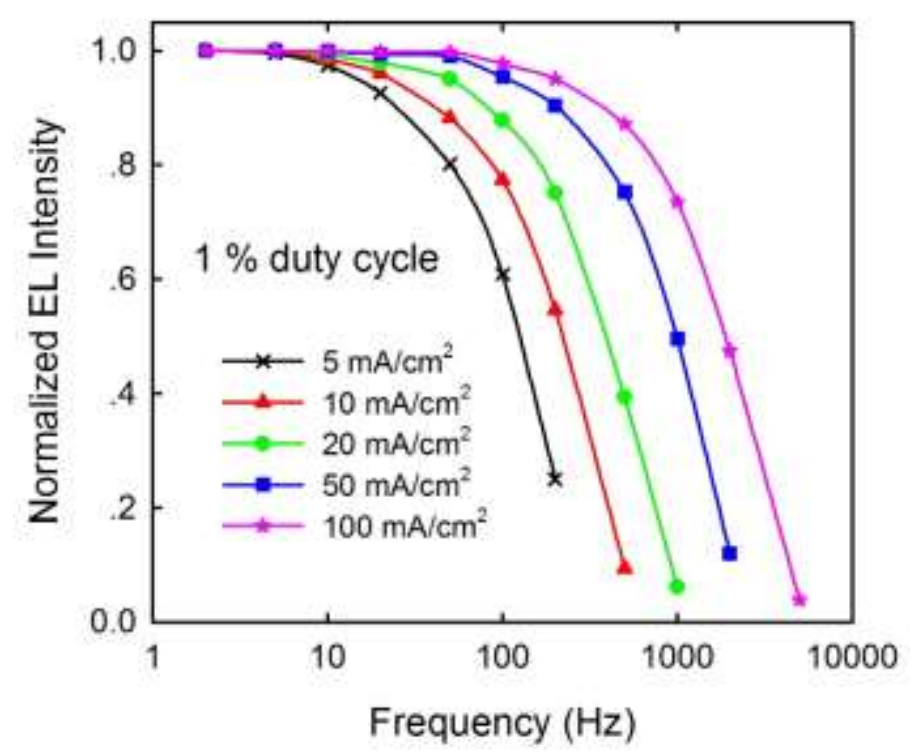

Figure 3.2. Normalized EL intensity as a function of pulse frequency and operating current level.

The duty cycle of the pulsed voltage is $1 \%$. The device structure is ITO / $\mathrm{MoO}_{3}$ / NPB / mCP:FIrpic (10 wt\%) / BPhen / LiF / Al.

In this chapter, a current density of $100 \mathrm{~mA} / \mathrm{cm}^{2}$ is used, which is much higher than the industrial standard. To largely eliminate the self-heating influence on the PhOLEDs reliability and, meanwhile, maintain a good brightness, the pulse frequency and the duty cycle are fixed at $100 \mathrm{~Hz}$ and $1 \%$, respectively. In this way, self-heating has a very short time to generate and long enough time to dissipate.

\subsection{Reliability of Blue PhOLEDs with different hosts}

Figure 3.3(a) shows the evolution of the normalized luminance of the blue PhOLEDs stressed at a constant continues wave (CW) current density of $100 \mathrm{~mA} / \mathrm{cm}^{2}$. At this current density, the PhOLEDs with the host of TCTA, CBP, mCP and UGH3 have initial luminance of 4105, 7372, 13839 and $2145 \mathrm{~cd} / \mathrm{m}^{2}$, respectively. Brightness of all devices exhibits fast initial decrease and typical stretched exponential decay. The OLED lifetime ( $\left.\mathrm{t}_{0.5}\right)$ is defined as the time lapsed for the OLED luminance at a constant current or voltage to decrease to half of its initial value. For the PhOLEDs with hosts of TCTA, CBP, mCP and UGH3, the lifetimes are 1.3, 8.6, 4.0 and $0.3 \mathrm{~min}$, respectively. 
Device degradation is accelerated when the device exhibits high luminance or undergoes electrical stressing with high current density. Because undesirable factors, such as self-heating, electro- and photo-chemical reactions, would give rise to defects at a considerable rate. Previous study showed that the degradation of electrically aged FIrpic emitters is mainly caused by (i) isomerization of the FIrpic molecules; and (ii) chemical dissociation of the FIrpic molecules by cleavage of the picolinate ligand [28]. The lifetime of PhOLED with CBP is 28 times as large as that with a UGH3 host under the same current density, implying that the host also has great impact on the OLED lifetime.

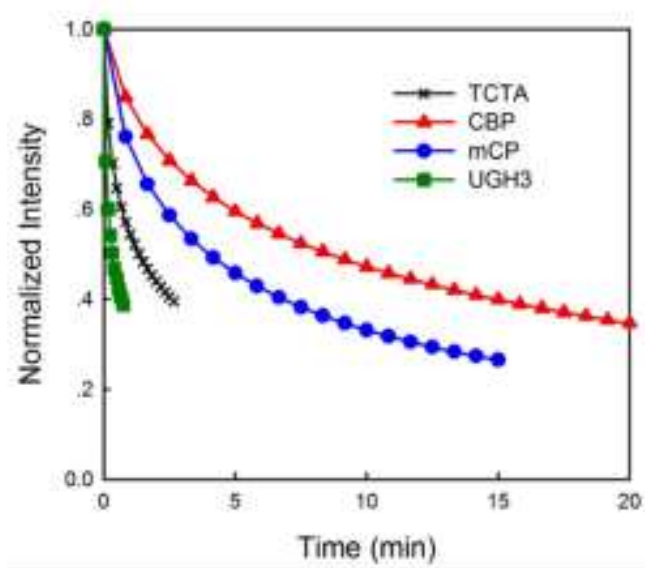

(a)

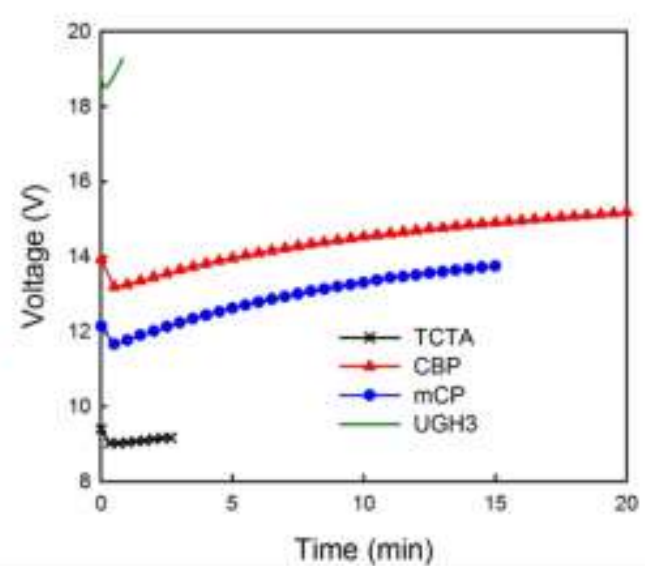

(b)

Figure 3.3. (a) Normalized luminance decay and (b) voltage evolution of blue PhOLEDs with different hosts stressed under CW $100 \mathrm{~mA} / \mathrm{cm}^{2}$.

The luminance decay is accompanied by a voltage rise, as shown in Fig. 3.3(b). All the devices shows an initial drop followed by steady rise over time. Normally, voltage rise is due to increasing defects in the OLED structure. Defects at layer interfaces and in the bulk of transport layers result in higher energy barrier and lower conductivity, respectively. The PhOLEDs with CBP and $\mathrm{mCP}$ show moderate rise rate, whereas the PhOLEDs with TCTA and UGH3 have mild and rapid rate, respectively. This fact may be attribute to the defect forming which is induced by self-heating. To maintain a constant current density, the PhOLEDs with different hosts have different operating voltage and therefore different overall input power. In Fig. 3.3(b), the device 
with host of UGH3 has largest power dissipation, which induces largest defect forming rate, leading to highest speed of voltage rise. This result is consistent with the fact that the UGH3 device suffers the shortest lifetime as well as the fastest degradation.

Figure 3.4 illustrates the current density-voltage $(\mathrm{J}-\mathrm{V})$ curves of representative fresh and stressed PhOLEDs on a semi-log scale. Some stressed devices exhibit increased low-biasing leakage current, whereas others show little change in leakage. This fact implies that the defects are generated in the device structure, forming continuous shunt leakage paths in some cases [29]. In the high injection regime, the current density of fresh device is higher than the stressed one at the same operation voltage. This difference is attributed to increase in series resistance, due to charge trapped at defect sites.

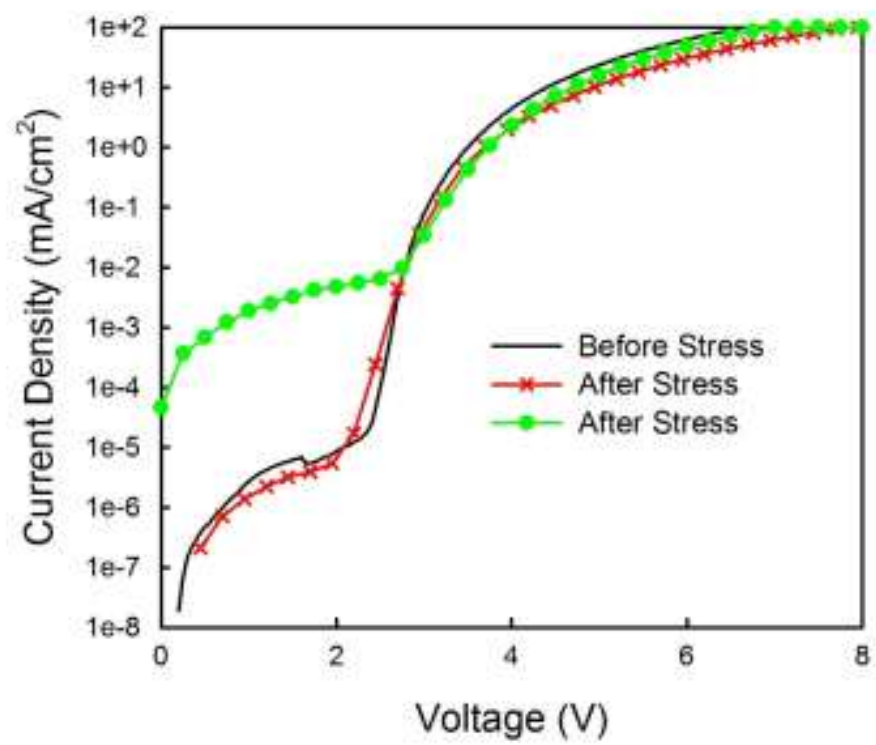

Figure 3.4. Current density-voltage characteristic of a representative PhOLED before and after stressing on a semi-log scale.

Figure 3.5 shows the evolution of the normalized luminance of the PhOLEDs stressed under $100 \mathrm{~Hz} 1 \%$ pulsed current density of $100 \mathrm{~mA} / \mathrm{cm}^{2}$. Like the decay curves under CW stressing conditions, all the PhOLEDs exhibit stretched exponential decay. The average values of $t_{0.5}$ of the PhOLEDs with host of TCTA, CBP, mCP and UGH3 are 410, 2330, 813, and 60 min, respectively, as shown in Table 3.1. Effective lifetime is defined as $\mathrm{t}_{0.5} \times$ duty cycle. For the $\mathrm{CW}$, the duty cycle is $100 \%$. Table 3.1 shows the 
effective lifetimes of the PhOLEDs under $\mathrm{CW}$ and pulsed stressing. The effective lifetimes under pulsed condition are found to be 2-3.2 times as large as $\mathrm{t}_{0.5}$ under $\mathrm{CW}$ condition. This result suggests that self-heating caused by current injection plays a role in luminance decay and device degradation in blue PhOLEDs. However, solely suppressing the thermal effect by pulsed injection does not result in long lifetime of the blue PhOLEDs. In particular, in the case of the PhOLED with UGH3, a significant amount of localized heating may be generated at the interface of hole transport layer (HTL) and emission layer (EML) due to a considerable energy barrier $(1.8 \mathrm{eV})$ at the interface. Pulsed operation extends the effective lifetime only by 2 times. Therefore, nonthermal factors must be responsible to the fast degradation of the PhOLEDs with UGH3, such as electrochemical reactions in the FIrpic molecules and undesired change in the host molecules. Since the blue PhOLEDs only differ in the host material, their different lifetimes imply different intrinsic factors of the host materials, for example, stability and charge mobility. For instance, UGH3 has very poor stability due to a low glass transition temperature $\left(\mathrm{T}_{\mathrm{g}}\right)$ of $46^{\circ} \mathrm{C}$, correlating with an extremely short lifetime of the PhOLED with UGH3. Under current stimulation, defects may be generated by the dissociation or crystallization of the host material, or reaction of the host molecules with FIrpic fragments. Since TCTA has a high $\mathrm{T}_{\mathrm{g}}\left(151^{\circ} \mathrm{C}\right)$, the PhOLEDs with TCTA are supposed to have a long lifetime. However, exciton recombination may occur in a very narrow zone in the EML near the electron transport layer (ETL), owing to the stark difference between electron and hole mobilities of TCTA $\left(\mu_{\mathrm{e}}<10^{-8} \mathrm{~cm}^{2} \mathrm{~V}^{-1} \mathrm{~s}^{-1}\right.$ and $\mu_{\mathrm{h}}=$ $3.0 \times 10^{-4} \mathrm{~cm}^{2} \mathrm{~V}^{-1} \mathrm{~s}^{-1}$ ) [30]. As a consequence, the PhOLEDs with TCTA suffer strong nonradiative recombination processes such as triplet-triplet annihilation (TTA) and triplet-polaron annihilation (TPA), which provide the major driving force for defect formation [31]. This assumption is validated by the low luminance of the PhOLEDs with TCTA, which is indicative of an inefficient radiative process. 


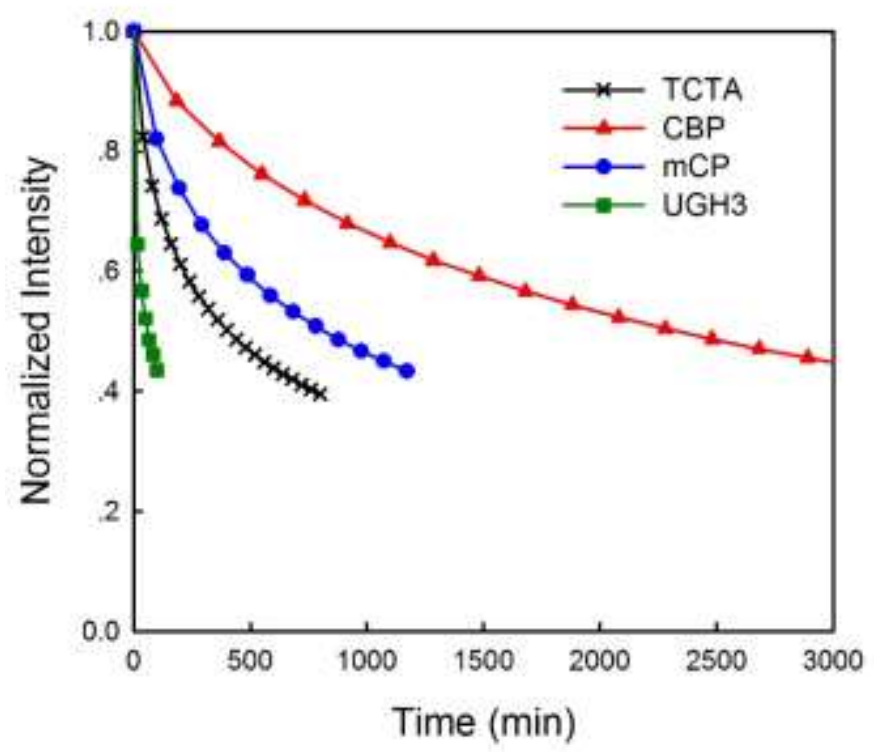

Figure 3.5. Normalized luminance decay of blue PhOLEDs with different hosts stressed under 100 $\mathrm{Hz}, 1 \%$, pulsed $100 \mathrm{~mA} / \mathrm{cm}^{2}$.

Table 3.1. Lifetimes of blue PhOLEDs with different hosts stressed under CW and pulsed condition $\left(100 \mathrm{~Hz}, 1 \%, 100 \mathrm{~mA} / \mathrm{cm}^{2}\right)$.

\begin{tabular}{|c|c|c|c|c|}
\hline & TCTA & CBP & $\mathrm{mCP}$ & UGH3 \\
\hline $\begin{array}{c}\mathrm{t}_{0.5}(\min ) \\
10 \mathrm{mACW}\end{array}$ & 1.3 & 8.6 & 4.0 & 0.3 \\
\hline $\begin{array}{c}\mathrm{t}_{0.5}(\mathrm{~min}) \\
10 \mathrm{~mA} 1 \%\end{array}$ & 483 & 2330 & 813 & 60 \\
\hline $\begin{array}{c}\mathrm{t}_{0.5}(\mathrm{~min}) \\
10 \mathrm{~mA} 1 \% \\
\text { (Effective) }\end{array}$ & 4.8 & 23.3 & 8.1 & 0.6 \\
\hline
\end{tabular}

The EL spectra help gain further insight into the degradation mechanisms. Figure 3.6 reveals slight color change (redshift) in the aged PhOLEDs as compared with asfabricated ones. The intensity ratio of the two peaks (502 nm and $474 \mathrm{~nm}$ ) for the PhOLEDs with TCTA changes from 0.99 before stressing to 1.04 after current stressing, as shown in Figure 3.6(a). It is worth noting that the spectra of both the unstressed and stressed devices were measured under the same current density and exposure time. As 
mentioned above, the hole mobility of TCTA is many orders of magnitude higher than the electron mobility, resulting in extremely unbalanced charge transport inside the EML. The recombination zone is very narrow located near the EML/ETL interface, where most defects are generated. As the defect traps are filled up by charge carriers, the recombination zone would be pushed away from the EML/ETL interface. Due to the interference effect, a redshift on the overall EL spectrum is expected. An even larger redshift has also been observed in the spectra of PhOLEDs with a CBP or mCP host, as shown in Figure 3.6(b). The intensity ratio of peaks at $502 \mathrm{~nm}$ and $474 \mathrm{~nm}$ changes from 1.00 to 1.25 after stressing. The larger ratio change indicate a larger shift of recombination zone toward the anode. As discussed in Chap. 2, PhOLEDs with a CBP or $\mathrm{mCP}$ host have a broader recombination zone as a result of more balanced charge transport. Therefore, defects would also be generated over a broader region, resulting in a larger shift of the recombination zone.

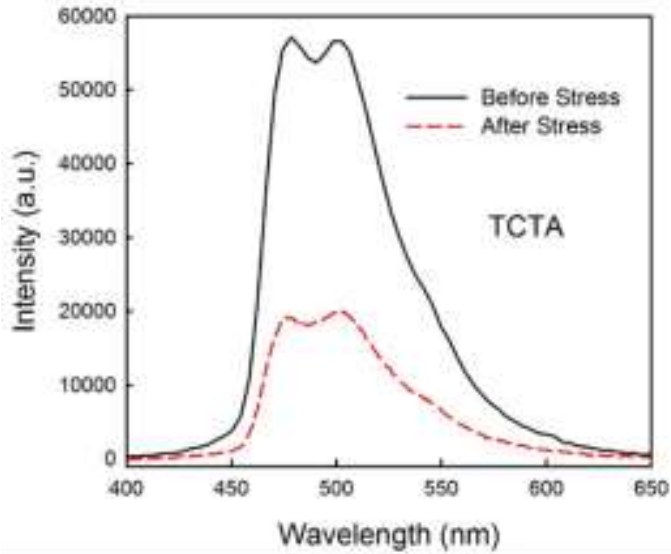

(a)

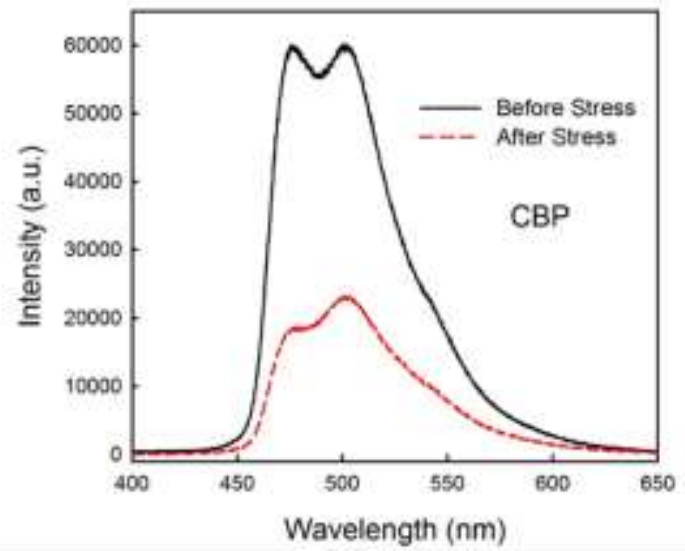

(b)

Figure 3.6. EL spectra of blue PhOLED with (a) TCTA and (b) CBP measured at $100 \mathrm{~mA} / \mathrm{cm}^{2}$ before and after current stressing.

Based on the above observation and analysis, three major material factors associated with the host would be identified to have significant impact on the reliability of blue PhOLEDs: (i) LUMO and HOMO energy levels, (ii) charge carrier mobility, and (iii) intrinsic stability. First, energetic misalignment of the host with the charge transport materials would result in an energy barrier for carrier injection and an 
additional voltage drop at interfaces, leading to localized joule heating and material degradation. Our study showed that suppression of the heating effect by pulsed stressing can improve the lifetime by 2-3.2 times. Second, mismatched electron and hole mobilities in the host cause unbalanced charge transport in the EML, leading to a narrow recombination zone. The above data showed that defects are mainly generated in such a narrow zone where most defects are generated. The recombination zone would be shifted if the defects are effective charge traps and are filled up during current injection. Finally, the host with poor intrinsic material stability can readily decompose under current stimulation or react with FIrpic fragments, forming charge traps and exciton quenchers. With a low $\mathrm{T}_{\mathrm{g}}$ of $46{ }^{\circ} \mathrm{C}, \mathrm{UGH} 3$ would not be thermally stable if temperature is elevated slightly above room temperature. This presumably explains the extremely short lifetime of blue OLEDs with FIrpic doped in a UGH3 host even under pulsed stressing.

\subsection{Investigation of other degradation factors}

\subsubsection{Reverse biasing}

To gain further insight into the degradation mechanisms of blue PhOLEDs, a reverse biasing $(-9 \mathrm{~V})$ was superimposed on the off cycle of the pulsed voltage source during the pulsed current stressing at $100 \mathrm{~mA} / \mathrm{cm}^{2}$. Figure 3.7 (a) illustrates the effective lifetimes of blue PhOLEDs with FIrpic doped in mCP under different stressing conditions. Improvement of lifetime by reverse biasing, from $8.1 \mathrm{~min}$ to $9.4 \mathrm{~min}$ was obtained. This improvement may be attributed to two possible effects of reverse biasing: (i) redistribution of mobile ions and accumulated charge carriers; and (ii) removal of defects acting as micro-channels. Ionic impurity and electric-field induced migration of mobile ions would otherwise provide quenching sites in the recombination zone in the EML, can be suppressed by the AC source. The accumulation of charge carriers at the interfaces and the charge traps, which are responsible for device degradation, can be released by a reverse bias. Zou et al. reported that a large reverse biasing results in a 
fast and large recovery of the device [32]. The reverse biasing can also remove defects, which may contribute to the leakage current. This can be seen in Fig. 3.7(b), the leakage current in low-bias region is reduced after stressing. The relatively small improvement (16\%) of the lifetime indicates that these effects play a minor role in the device degradation.

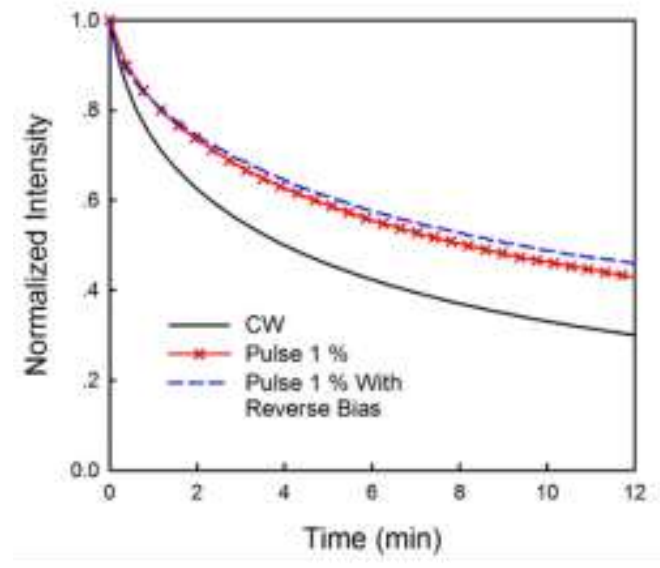

(a)

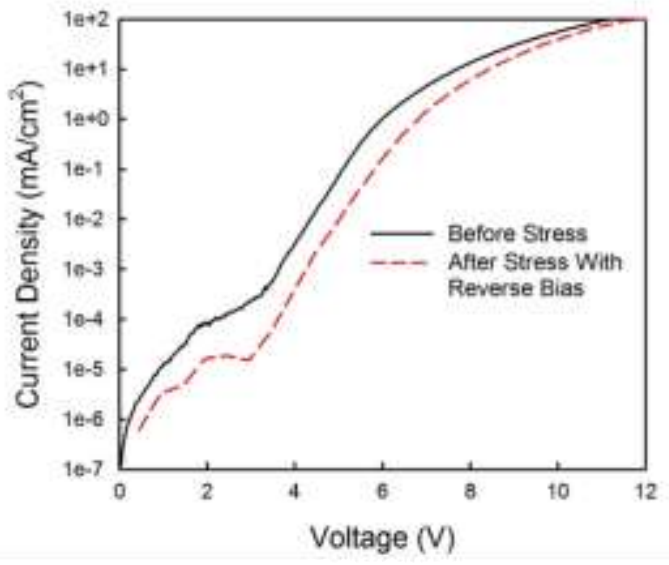

(b)

Fig. 3.7. (a) Effective lifetimes of blue PhOLEDs measured with current density of $100 \mathrm{~mA} / \mathrm{cm}^{2}$ CW, pulsed with and without a reverse biasing. (b) Current density-voltage characteristics of blue PhOLEDs before and after pulsed stressing with a reverse biasing.

\subsubsection{Doping concentration}

To determine if the doping concentration of FIrpic plays a role in the device aging process, PhOLEDs with 4.2-14.6wt\% FIrpic doped in $\mathrm{mCP}$ were fabricated and subjected $10 \mathrm{~mA} / \mathrm{cm}^{2}$ stressing. Figure 3.8 shows the luminance evolution of these blue PhOLEDs. The parasitic effects like TTA and TPA, can cause molecule decomposition and device degradation [31]. These effects should be less pronounced at low doping levels, and thus, the PhOLEDs with low doping concentrations would have a longer lifetime. However, as shown in Fig. 3.8, the device lifetime increases with the doping concentration. Compared with $4.2 \mathrm{wt} \%$, the device with doping concentration of 14.6 wt\% improves the lifetime by 2.5 times. This confliction can be explained by considering the energy level diagram and charge transport behavior of the EML. mCP is an ambipolar host material, but it favors hole transport, causing unbalanced charge 
transport within the host. However, addition of FIrpic molecules can enhance electron injection and transport as its LUMO level aligns well with that of BPhen. Especially at a high doping concentration, electrons can inject directly onto the FIrpic molecules and move via hopping. As a result, the hole and electron currents are more balanced within the EML, leading to a broader recombination zone [33]. Indeed, at $20 \mathrm{~mA} / \mathrm{cm}^{2}$, the voltage of the PhOLED with $14.6 \%$ FIrpic is $1.2 \mathrm{~V}$ smaller compared to that of the $4.2 \%$ device. It is believed that the smaller voltage and broader recombination zone are responsible for the extended lifetime at high FIrpic concentrations.

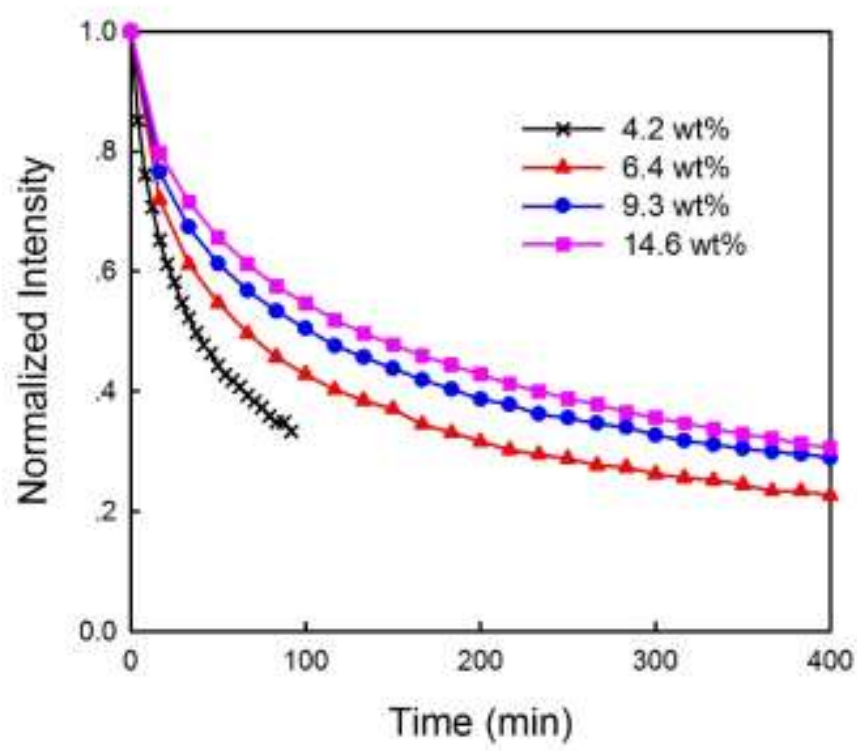

Fig. 3.8. Normalized luminance decay of blue PhOLEDs with different weight ratios under CW 10 $\mathbf{m A} / \mathbf{c m}^{2}$.

\subsubsection{Post thermal treatment}

Furthermore, post thermal treatment of the PhOLEDs with an mCP host was conducted and its possible influence on the device lifetime was investigated. After deposition of all the layers, annealing at a variety of temperatures was conducted in $\mathrm{N}_{2}$ filled glove box, followed by encapsulation. The thermally treated devices show improved J-V curves, as shown in Figure 3.9(a). At a given current density, the operating voltage decreases with annealing temperature. At the current density of 100 $\mathrm{mA} / \mathrm{cm}^{2}$, the operating voltage of untreated devices is $11.4 \mathrm{~V}$, whereas for the device 
annealed at $60{ }^{\circ} \mathrm{C}$ it is $9.0 \mathrm{~V}$. This improvement may be attributed to more intimate contact among different organic layers as well as between the organic layers and the electrodes. It was reported that interdiffusion of the organic layers was observed when the annealing temperature was over $100{ }^{\circ} \mathrm{C}$ [34]. In our experiment, the highest temperature was $60{ }^{\circ} \mathrm{C}$, much lower than $100{ }^{\circ} \mathrm{C}$. Therefore, interdiffusion may play a minor role in this scenario.

Meanwhile, also it was found that the treatment also affected the brightness. Figure 3.9(b) illustrates the brightness of blue PhOLED measured after treatment at different temperatures. The brightness maintains a stable level when the annealing temperature is no more than $50{ }^{\circ} \mathrm{C}$, whereas the brightness declines abruptly when the temperature is over $50{ }^{\circ} \mathrm{C}$. The device failure above $50{ }^{\circ} \mathrm{C}$ is attributed to the poor thermal stability of the organic materials. For example, the $\mathrm{T}_{\mathrm{g}}$ of $\mathrm{mCP}$ is only $55^{\circ} \mathrm{C}$.

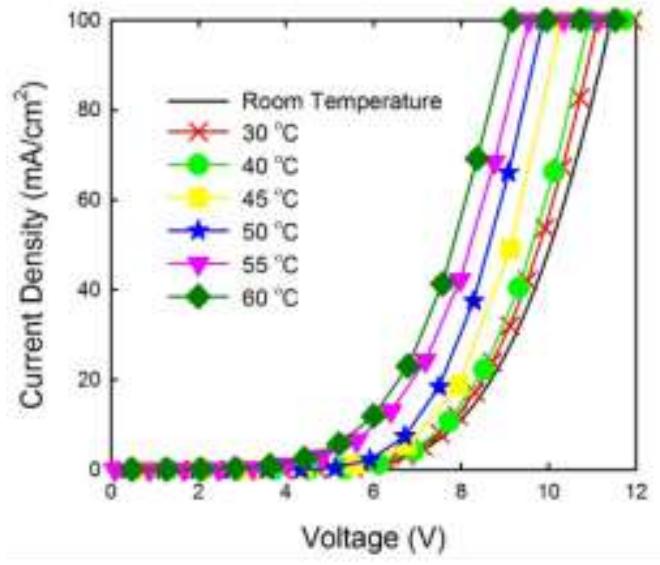

(a)

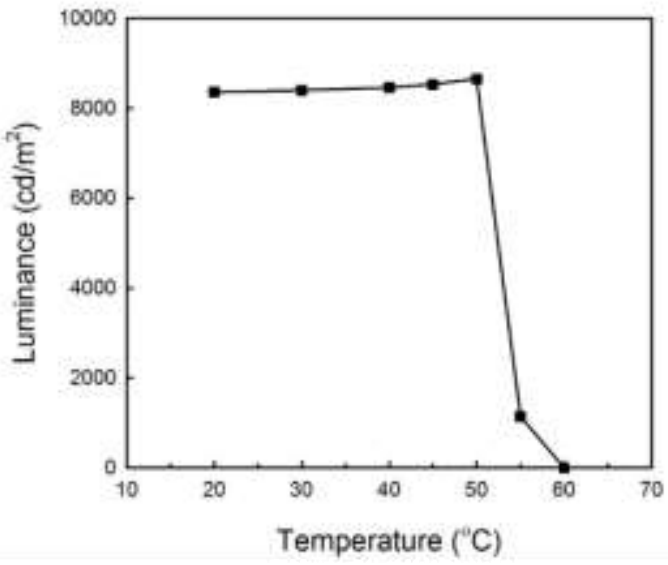

(b)

Fig. 3.9. (a) Current density-voltage characteristics and (b) brightness of blue PhOLEDs annealed at a variety of temperatures. The host material is $\mathrm{mCP}$.

The same treatment was also done with blue PhOLEDs with a TCTA host. Figure 3.10(a) shows the operating voltage at current density of $100 \mathrm{~mA} / \mathrm{cm}^{2}$ as a function of the annealing temperature. The operating voltage decreases slowly, followed by a fast decline above $80^{\circ} \mathrm{C}$. It is plausible that above $80{ }^{\circ} \mathrm{C}$, the interdiffusion of organic layers becomes pronounced, leading to lower energy barriers. Interdiffusion may also be responsible for the brightness quenching, as shown in Figure 3.10(b). The PhOLED 
with TCTA appears to be more thermally stable than that with $\mathrm{mCP}$, probably due to the higher $\mathrm{T}_{\mathrm{g}}$ of TCTA $\left(151^{\circ} \mathrm{C}\right)$. This result suggests that the thermal property of the host material may have a significant impact on the thermal stability of blue PhOLEDs.

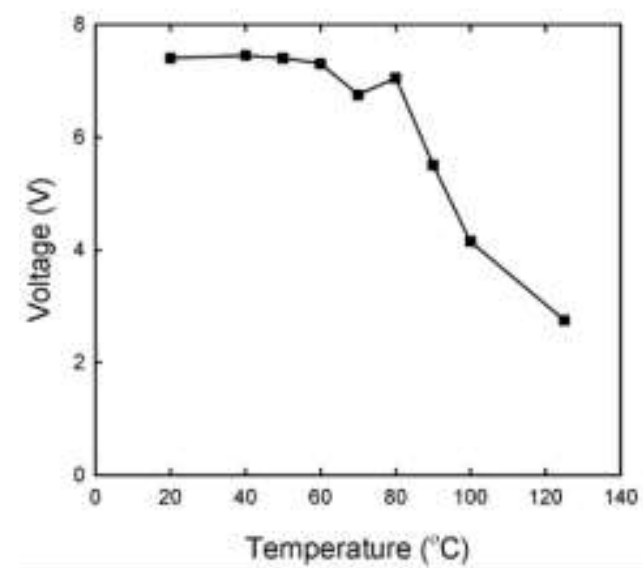

(a)

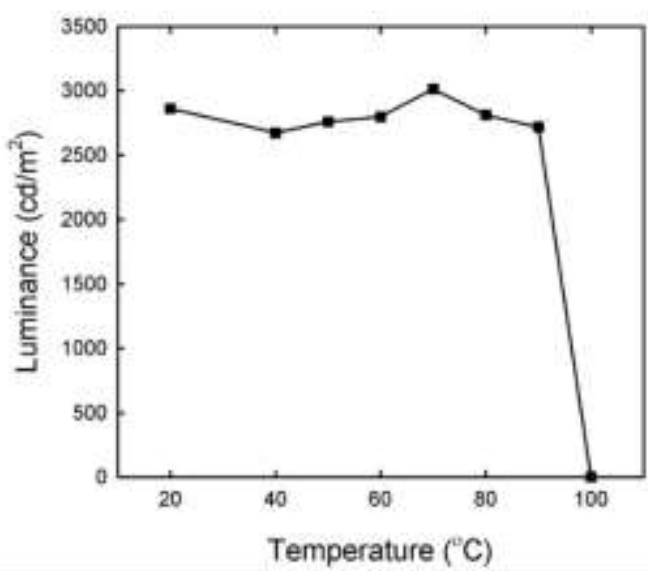

(b)

Fig. 3.10. (a) Operating voltage and (b) luminance change with annealing temperature. The host material is TCTA.

Figure 3.11(a) shows the luminance evolution of blue PhOLEDs with mCP host annealed at different temperatures and stressed at $100 \mathrm{~mA} / \mathrm{cm}^{2}$. The lifetime does not change when the devices annealed below $45{ }^{\circ} \mathrm{C}$, whereas the device annealed at $50{ }^{\circ} \mathrm{C}$ shows a lifetime improved by $70 \%$. We attributed the longer lifetime to improved charge transport across interfaces and better morphology in the thermally treated PhOLEDs [34].

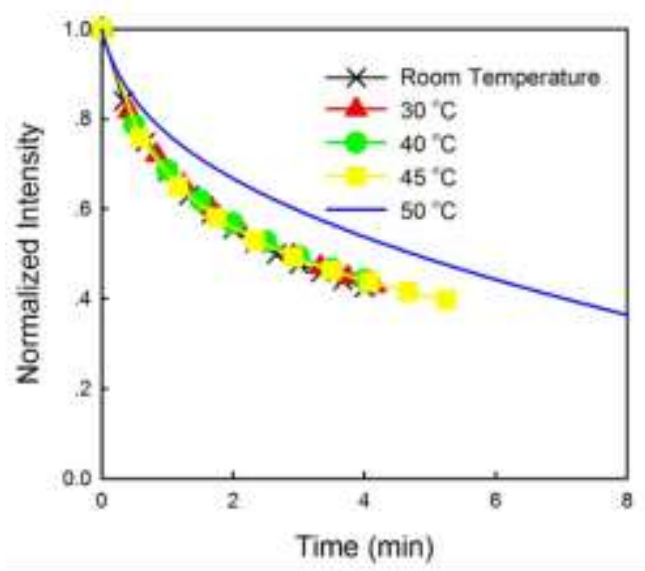

(a)

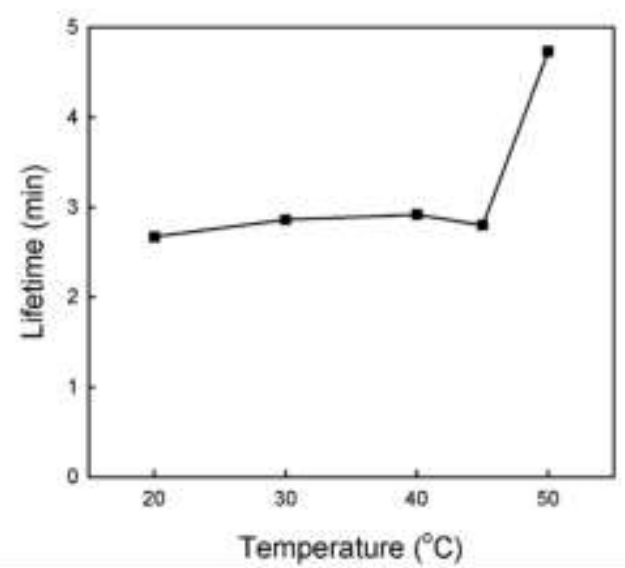

(b) 
Fig. 3.11. (a) Normalized luminance decay and (b) lifetime of blue PhOLEDs annealed at different temperature under CW stressing.

\subsection{Conclusions}

Degradation of blue PhOLEDs based on FIrpic doped in different hosts was studied. The devices were stressed by continuous and pulsed source at a constant current density of $100 \mathrm{~mA} / \mathrm{cm}^{2}$, which is much higher than industrial standard. The PhOLEDs exhibited rapid stretched exponential decay of luminance with lifetime varied from 0.3-8.6 min, evidencing an important role of the host in the OLED degradation process. The device degradation was accompanied by voltage rise, due to the defect accumulation at interfaces and in the bulk of organic layers. Stressing with $1 \%$ pulsed current extended the lifetime only by 2-3.2 times. The EL spectra of aged devices with a host favoring hole transport showed a red shift, implying that the main recombination zone was pushed away from the EML/ETLinterface. The effects of other aging factors, including reverse biasing, doping concentration, and post thermal treatment were also studied. However, the resulting improvements were limited, and the optimized blue PhOLED still showed very poor reliability under current stressing. These findings suggested that fast degradation of typical blue PhOLEDs is mainly caused by current-induced electrochemical reactions in the host and emitter materials within a narrow recombination zone. These results also stress the importance of selecting an appropriate host for reliable operation of blue phosphorescent OLEDs, which should have good stability, enable balanced and efficient charge injection and transport within the EML.

\section{References}

[1] L. Xiao, Z. Chen, B. Qu, J. Luo, S. Kong, Q. Gong and J. Kido, Adv. Mater. 23, 926-952 (2011). 
[2] K. S. Yook and J. Y. Lee, Adv. Mater. 24, 3169-3190, (2012).

[3] W. C. H. Choy, W. K. Chan and Y. Yuan, Adv. Mater. 26, 5368-5399 (2014).

[4] H. Xu, R. Chen, Q. Sun, W. Lai, Q. Su, W. Huang and X. Liu, Chem. Soc. Rev. 43, 3207-3812, (2014).

[5] H. Sasabe and J. Kido, Eur. J. Org. Chem., 7653-7663 (2013).

[6] X. Yang, X. Xu and G. Zhuo, J. Mater. Chem. C, 3, 913, (2015).

[7] S. Scholz, D. Kondakov, B. Lussem and K. Leo, Chem. Rev., 115, 8449-8503, (2015).

[8] Y. Zhang, J. Lee and S. R. Forrest, Nat. Comm. 5, 5008 (2014).R. Seifert, I. R. de Moraes, S. Scholz, M. C. Gather, B. Lussem and K. Leo, Org. Electron. 14, 115123, (2013).

[9] M. C. Gather and S. Reineke, J. Phot. Eng. 5, 057607-1, (2015).

[10] H. Jeong, H. Shin, J. Lee, B. Kim, Y. Park, K. S. Yook, B. K. An and J. Park, J. Phot. Eng. 5, 057608-1, (2015).

[11] J. Wang, F. Zhang, J. Zhang, W. Tang, A. Tang, H. Peng, Z. Xu, F. Teng and Y. Wang, Journal of Photochemistry and Photobiology C: Photochemistry Reviews, 17, 69-104, (2013)

[12] L. S. Hung and C. H. Chen, Mate. Sci. Eng. R, 39, 143-222, (2002).

[13] G. Zhou, W. Y. Wong and X. Yang, Chem. Asian J., 6, 1706-1727, (2011).

[14] T. Tsuboi, Journal of Non-Crystalline Solids, 356, 1919-1927, (2010).

[15] S. W. Wen, M. T. Lee and C. H. Chen, IEEE/OSA Journal of Display Technology, 1, 90, (2005).

[16] C. Ulbricht, B. Beyer, C. Friebe, A. Winter, and U. S. Schubert, Adv. Mater., 21, 4418-4441, (2009).

[17] B. Mi, Z. Gao, Z. Liao, W. Huang and C. H. Chen, Sci. China Chem., 53, 16791694, (2010).

[18] S. Schmidbauer, A. Hohenleutner and B. Konig, Adv. Mater., 25, 2114-2129, (2013). 
[19] F. So and D. Kondakov, Adv. Mater., 22, 3762-3777, (2010).

[20] S. Scholz, D. Kondakov, B. Lussem and K. Leo, Chem. Rev., 115, 8449-8503, (2015).

[21] H. Aziz and Z. D. Popovic, Chem. Mater., 16, 4522-4532, (2004).

[22] J. Wang, F. Zhang, J. Zhang, W. Tang, A. Tang, H. Peng, Z. Xu, F. Teng and Y. Wang, Journal of Photochemistry and Photobiology C: Photochemistry Reviews, 17, 69-104, (2013).

[23] L. S. Hung and C. H. Chen, Mate. Sci. Eng. R, 39, 143-222, (2002).

[24] N. Grossiord, J. M. Kroon, R. Andriessen and P. W. M. Blom, Organic Electronics, $13,432-456,(2012)$.

[25] Z. D. Popovic and H. Aziz, IEEE Journal on Selected Topics in Quantum Electronics, 8, 362, (2002).

[26] D. G. Moon, R. B. Pode, C. J. Lee, and J. I. Han, App. Phys. Lett., 85, 4771 (2004).

[27] V. K. Chandra and B. P. Chandra, Org. Electron., 13, 329-334, (2012).

[28] I. R. de Moraes, S. Scholz, B. Lussem and K. Leo, Org. Electron., 12, 341-347, 2011.

[29] X. Li and X. Cao, Org. Electron., 14, 2523-2527, (2013).

[30] J. W. Kang, S. H. Lee, H. D. Park, W. I. Jeong, K. M. Yoo, Y. S. Park, and J. J. Kim, Appl. Phys. Lett. 90, 223508 (2007).

[31] N. C. Giebink, B. W. D’Andrade, M. S. Weaver, P. B. Mackenzie, J. J. Brown, M. E. Thompson, and S. R. Forrest, App. Phys. Lett., 103, 044509 (2008).

[32] M. Yahiro, D. Zuo and T. Tsutsui, Synthetic Metals, 111-112, 245-247, (2000).

[33] T. L. Chiu and P. Y. Lee, Int. J. Mol. Sci., 13, 7575-7585, (2012).

[34] G. T. Chen, S. H. Su, C. C. Hou and M. Yokoyama, Journal of the Electrochemical Society, 154, J159-J162, (2007). 


\section{Chapter 4 Conclusions and Future Work}

\subsection{Conclusions}

This thesis work aimed at fabrication and reliability study of blue PhOLEDs with FIrpic doped in different wide bandgap hosts. The goal is to gain a greater understanding of the impact of the host material on the device reliability and the underlying degradation mechanisms of the devices subjected to current stressing under different conditions. The major results and conclusions are summarized below:

(i) The baseline blue PhOLED with FIrpic doped in an mCP host was fabricated. Thin layers of $\mathrm{MoO}_{3}$ and $\mathrm{LiF}$ were inserted between the electrodes and organic structure to enhance hole and electron injection, respectively. The thickness of the BPhen ETL was tailored in order to obtain the highest brightness and the optimal thickness was found to be $\sim 45 \mathrm{~nm}$.

Blue PhOLEDs with FIrpic doped in four different host materials were fabricated and compared. The PL spectra of all the hosts overlapped the absorption spectrum of FIrpic, indicating good energy transfer from the host to FIrpic. PhOLEDs with mCP had the highest luminance and the highest external quantum efficiency due to three factors: favorable energy level alignment, good triplet energy confinement and balanced electron/hole transport. At $20 \mathrm{~mA} / \mathrm{cm}^{2}$, the voltage was $7.9 \mathrm{~V}$, and a luminance of $3320 \mathrm{~cd} / \mathrm{m}^{2}$ was obtained. In contrast, the PhOLEDs with a TCTA or UGH3 host exhibited low luminance and efficiency mainly due to unbalanced charge injection and transport within the EML.

(ii) Degradation of blue PhOLEDs based on FIrpic doped in different hosts was studied. The devices were stressed by continuous and pulsed source at a constant current density of $100 \mathrm{~mA} / \mathrm{cm}^{2}$, which is much higher than industrial standard. The PhOLEDs exhibited rapid stretched exponential decay of luminance with lifetime variation from 0.3-8.6 min, evidencing an important role of the host in the OLED degradation process. The device degradation was accompanied by voltage rise, due to 
the defect accumulation at interfaces and in the bulk of organic layers. Stressing with $1 \%$ pulsed current extended the lifetime only by 2-3.2 times. The EL spectra of aged devices with a host favoring hole transport showed a red shift, implying that the main recombination zone was pushed away from the EML/ETL interface. The effects of other aging factors, including reverse biasing, doping concentration, and post thermal treatment were also studied. However, the resulting improvements were limited, and the optimized blue PhOLED still showed very poor reliability under current stressing. These findings suggested that fast degradation of typical blue PhOLEDs is mainly caused by current-induced electrochemical reactions in the host and emitter materials within a narrow recombination zone. These results also stress the importance of selecting an appropriate host for reliable operation of blue phosphorescent OLEDs, which should have good stability, enable balanced and efficient charge injection and transport within the EML.

\subsection{Future work}

The state-of-the-art blue PhOLEDs are still far from mature and suffer from poor reliability. Further research work needs to be conducted to understand key limiting intrinsic factors and improve the device lifetime at high luminance levels. While it is important to synthesize new blue phosphorescent materials with high efficiency as well as good stability, our research showed that it is also vital to seek an appropriate host for a specific emitter to achieve reliable operation of the PhOLED. The host material should be energetically aligned with the charge transport materials, have good stability, and enable balanced and efficient charge injection and transport.

In this work, we did not intentionally add electron and hole blocking layers in the PhOLED structure, which would greatly improve exciton confinement in the EML and the device luminous efficiency. How these charge blocking layers affect the lifetime of blue PhOLEDs is a topic worth studying. 